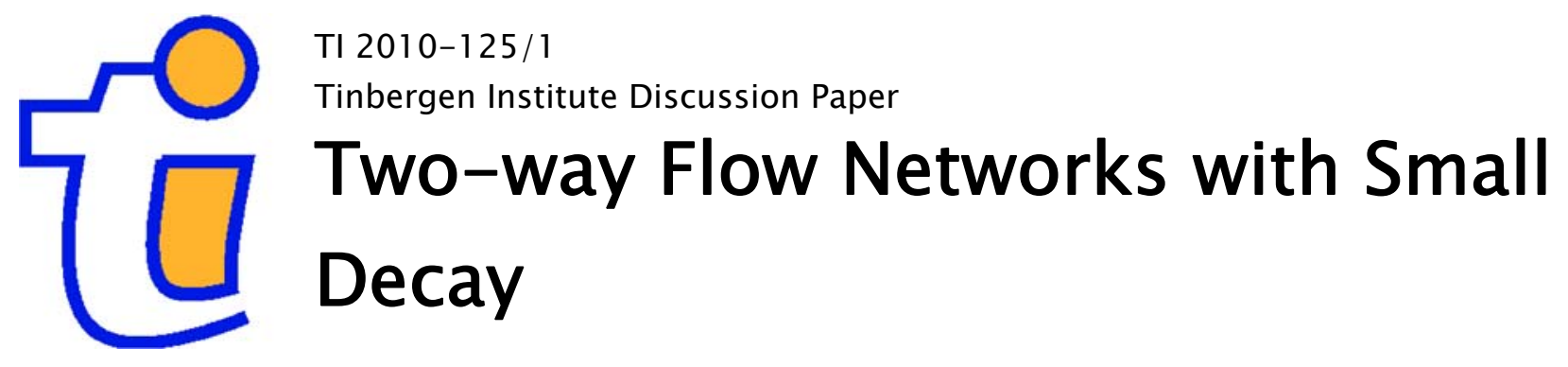

K. De Jaegher

J.J.A. Kamphorst ${ }^{2}$

' Utrecht School of Economics, Utrecht University, the Netherlands;

2 Erasmus School of Economics, Erasmus University Rotterdam, the Netherlands 


\section{Tinbergen Institute}

The Tinbergen Institute is the institute for economic research of the Erasmus Universiteit Rotterdam, Universiteit van Amsterdam, and Vrije Universiteit Amsterdam.

Tinbergen Institute Amsterdam

Roetersstraat 31

1018 WB Amsterdam

The Netherlands

Tel.: +31(0)205513500

Fax: $+31(0) 205513555$

Tinbergen Institute Rotterdam

Burg. Oudlaan 50

3062 PA Rotterdam

The Netherlands

Tel.: + $31(0) 104088900$

Fax: $+31(0) 104089031$

Most TI discussion papers can be downloaded at http://www.tinbergen.nl. 


\title{
Two-way flow networks with small decay
}

\author{
K. De Jaegher \\ Utrecht School of Economics \\ Utrecht University \\ k.dejaegher@uu.nl
}

\author{
J.J.A. Kamphorst \\ Erasmus School of Economics \\ Erasmus University Rotterdam \\ kamphorst@ese.eur.nl
}

\section{Work in progress: November $2010^{1}$}

\begin{abstract}
This paper characterizes the set of equilibrium networks in the twoway flow model of network formation with small decay, and this for all increasing benefit functions of the players. We show that as long as the population is large enough, this set contains large- as well as small-diameter networks. For all benefit functions, the periphery-sponsored star is the most stable. When the marginal benefits of information are constant, all non-star networks are equally stable. With increasing marginal benefits of information, small-diameter networks in general tend to be more stable. However, with decreasing marginal benefits of information, large-diameter networks tend to be the most robust along with the periphery-sponsored star.
\end{abstract}

\section{Introduction}

In network formation models, information decay reflects the intuition that information becomes less valuable the further it has to travel through the network. ${ }^{2}$ Decay has two effects on network formation. First, ex-ante, homogeneous players become heterogeneous by their position in the network. A player with many direct links in the network, or a player who forms a bridge in the network through which information between many other players travels, may be more attractive to connect to. Second, decay may give the individual player an incentive to sponsor links to players from which he already indirectly receives

\footnotetext{
${ }^{1}$ This paper arose from comments by Ewa Mendys, and we are grateful to her. We also thank Corinne Autant-Bernard, Pascal Billand, Christophe Bravard, Berno Büchel, Vincent Buskens, Rense Corten, Marco van der Leij, Jacqueline Morgan, Stephanie Rosenkranz, Klaas Staal and Bastian Westbrock for valuable comments, as well as the participants of the ISDG13 in Wroclaw, the $5^{\text {th }} \mathrm{SFB} / \mathrm{TR} 15$ workshop for young researchers in Berlin, the $7^{\text {th }}$ workshop on networks in economics and sociology: dynamic networks in Utrecht, the LSU Conference on 'Networks: Theory and Applications', the $15^{\text {th }}$ CTN workshop in Marseille, the EEA conference in Glasgow, and a CREUSET seminar in St.Etienne.

${ }^{2}$ For example, consider a situation in which an agent benefits from connections to other agents because other agents might hear information (say on job openings, or sales) which this agent has missed. Having a connection to other agents allows the agent to benefit from the information which other players receive. Of course, information may be passed on by third agents. As communication is typically imperfect, one could say that more of the information is lost when it has to be passed on through more other agents. This is an example of decay.
} 
information; if these players are so distant that their information has decayed a lot, it may still be of interest to the individual player to also connect directly to them. The second effect only exists for large enough levels of decay, but the first effect exists for all positive levels of decay. It follows that it is possible to analyze the first effect in isolation, which is the purpose of this paper. Roughly speaking, we say that there is small decay when the second effect does not play a significant role.

We use the two-way flow model of network formation, as introduced in the seminal Bala and Goyal (2000a). ${ }^{3}$ The authors provide a full characterization of the set of strict Nash networks (SNNs) - so where every player strictly prefers to keep his current strategy - for the case where there is no decay. They show, for very general payoff functions, that there is just a single strict Nash network architecture, namely the center-sponsored $\operatorname{star}^{4}$. However they make another important point. By means of a few specific examples in a setting with linear benefits, they show that the set of strict Nash network architectures becomes much richer when there is small decay. These examples are all small-diameter networks, so in those example networks all players are close to each other. Bala and Goyal (2000a) do not further investigate the necessary and sufficient structural properties which strict Nash networks will satisfy under decay. Still, it seems then that the general result of the stability of small-diameter networks is maintained. This intuition is confirmed in Hojman and Szeidl's (2008) extension of the two-way flow model to the case of decay. They find that the unique SNN architecture is the periphery-sponsored $\operatorname{star}^{5}$ if $(i)$ the population is large enough; (ii) there is a maximal distance at which information can flow; (iii) there is decay ${ }^{6}$; and $(i v)$ the benefit function is concave enough.

The two-way flow model has been extensively studied and has been modified in several directions such as player heterogeneity ${ }^{7}$, link reliability ${ }^{8}$ and non-linear benefits ${ }^{9}$. In contrast to many of those papers, we do not extend

\footnotetext{
${ }^{3}$ In that network, the agents (who are the nodes) decide to which of the other agents they sponsor links. This generates a network. Agents have incentives to sponsor links because they receive some benefit for each agent that they are connected to via a series of links (a path). On the other hand, sponsoring links is costly. In their basic model the benefits which a player derives from being connected to some other agent only depends on whether there is a connection, not on the length of the path.

${ }^{4} \mathrm{~A}$ network where one player sponsors a link to each other player, while the other players do nothing.

${ }^{5} \mathrm{~A}$ network in which there is a unique player who sponsors no links, say player $i$, and where all other players sponsor one link each, namely to player $i$.

${ }^{6}$ Their decay function is a very nice generalization of the typical decay function which we will use.

${ }^{7}$ For instance, Billand et al. (2006), Galeotti et al. (2006) and Kamphorst and Van der Laan (2007).

${ }^{8}$ See for instance Bala and Goyal (2000b), Haller et al. (2005) and Billand et al. (2006).

${ }^{9}$ See Vergara-Caffarelli (2004) for an example in the one-way flow model; Buechel (2007) for an example with two sided link formation; Goyal and Joshi (2006) for an example with two-sided link formation and non-linearity of payoffs in the number of own links and the number of links by others; and Bloch and Dutta (2009) for an example with endogenous link strength (and non-linearity of benefits in link strengths).

Note also that, for the case without decay, Bala and Goyal (2000a) adopts a very general
} 
the two-way flow model, but instead we continue the analysis of decay where it stopped in Bala and Goyal (2000a). Specifically, the contribution of our paper is a full characterization of strict Nash networks with decay. Contrary to Hojman and Szeidl (2008), we do not consider an exogenously given maximal distance at which information can flow, and we do not just consider decreasing marginal benefits of information, but any benefit function that is increasing in information. We focus on small decay, so that any minimal connected network potentially is a strict Nash network, or such that the set of strict Nash networks does not contain non-minimal networks.

Based on the simple principle that in a strict Nash network, every sponsor of a link should connect to a unique best-informed player in the accessed component, we are able to show that minimal connected networks that are candidate strict Nash networks can be divided into two groups, namely networks with a unique characterizing player who receives multiple links, and networks with a unique characterizing player who does not receive any links. In both types of candidate networks, with the exception of the links directly sponsored to the characterizing player, the direction of sponsoring points away from the characterizing player. The characterizing player is either the best-informed player in the network, or sponsors a link to the best-informed player in the network. In general, the more distant in the network a player is from the characterizing player, the less information he has.

In any minimal connected network that is a potential strict Nash network, each recipient of a link either sponsors no links, or sponsors at least two links. In combination with the result that the direction of sponsoring tends to point away from the characterizing player, this shows that as long as the population is large enough, the diameter of a candidate strict Nash network can be arbitrarily large.

We next importantly show that parameters exist such that the set of candidate strict Nash networks, i.e. the set of minimal connected networks that meet the necessary conditions that we have listed so far, indeed fully characterizes the set of strict Nash networks. The characterization is completed by deriving, for small decay, a condition which we refer to as the balancing condition, and which ensures that the architecture of the network is such that each sponsoring player sponsors a link to the unique best-informed player in the component accessed. The balancing condition further allows us to characterize the maximal-diameter networks for a specific set of populations, and to confirm that this maximal diameter is arbitrarily large, as long as the population is sufficiently large.

We further investigate, for specific forms of the benefit function, the stability of the set of strict Nash networks to increases in linking costs. For all benefit functions, the architecture where all players sponsor a single link to a single central player (periphery-sponsored star) is the most stable. Intuitively, the benefit of sponsoring a link to a peripheral player in such a network is large, as the choice lies between having no information at all, or having all players close by, thus obtaining high-quality information.

payoff function which is in no way restricted to linear benefits. 
Further stability results depend on the form of the benefit function. With constant marginal benefits of information, all networks that meet our necessary conditions and are not periphery-sponsored stars, are equally stable. Under increasing marginal benefits of information, small-diameter networks tend to be more stable. Intuitively, with increasing marginal benefits of information, players have more incentives to sponsor links the more information they already have, i.e. the closer they already are to other players in the network. This makes small-diameter networks more stable.

Under decreasing marginal benefits, however, with the exception of the periphery-sponsored stars, large-diameter networks tend to be more stable. Intuitively, with decreasing marginal benefits of information, players have more incentives to sponsor links the less information they already have, i.e. the further away they are from other players in the network. This makes large-diameter networks more robust. In a general version of this result, we show that a gap exists in the diameters covered by the strict Nash networks. Further, for a specific example, we show that cost levels exist such that the only strict Nash networks are the periphery-sponsored stars, and the networks with maximal diameter, or with diameter one unit lower than the maximal diameter. These results go radically against the intuition that small-diameter networks are more stable, reflected by results presented in Bala and Goyal (2000a), and Hojman and Szeidl (2008).

The paper is structured in such a manner that we start by deriving a maximum number of results for a minimal number of assumptions. Further assumptions, in particular about the form of the benefit function and the level of decay, are introduced later on to make the characterization more detailed. In Section 2 we present the game, introduce terminology and notation, and derive some standard preliminary results. Section 3 characterizes the necessary structural properties of mimimal connected SNNs, and this for a general increasing benefit function. The relative stability of different candidate SNN minimal connected networks is then studied in Section 4 for three special types of benefit functions: concave, linear and convex. We are able to extend the characterization further in Section 5 by looking at levels of decay which are sufficiently small. This results in a condition which we call the balancing condition. This balancing condition allows us to show that all networks which satisfy the balancing condition as well as the properties derived in Section 3 are indeed SNNs for some positive range of the parameters. Hence each such network is indeed relevant. Further, we use the balancing condition to show that, as long as the population is large enough, there is no upper limit on the maximal diameter that a strict Nash network may have. Moreover, under concave benefits of information, we provide an example where for high costs, the only stable networks are the periphery-sponsored star and the candidate networks with the largest diameters. Section 6 concludes with a discussion of the results. 


\section{The Model}

In this section we first present the game (Section 2.1), then we introduce some useful notation and terminology, and two important architectures (Section 2.2). We end this section by providing some standard results regarding the existence and architecture of strict Nash networks (Section 2.3).

\subsection{The game}

Let $\mathcal{N}$ be the set of players with cardinality $n$, where $n \geq 3$. Each player faces the choice which of the other players he will sponsor a link to. A link by player $i$ (the sponsor) to player $j$ (the recipient) is denoted by $i j$. The set of all links that a player $i$ can possibly sponsor is given by $\mathcal{L}_{i} \equiv\{k j \in \mathcal{N} \times \mathcal{N}: k=i, j \neq i\}$. $\mathcal{L}$ is defined as the set of all possible links, meaning that $\mathcal{L} \equiv \bigcup_{i \in \mathcal{N}} \mathcal{L}_{i}=$ $\{i j \in \mathcal{N} \times \mathcal{N}: i \neq j\}$. We typically denote the strategy of player $i-$ the set of links that he sponsors - by $g_{i}$. His strategy space $\mathcal{G}_{i}$, where obviously $g_{i} \in \mathcal{G}_{i}$, is therefore the collection of all subsets of $\mathcal{L}_{i}$, specifically $\mathcal{G}_{i} \equiv\left\{g_{i} \subseteq \mathcal{N} \times \mathcal{N}: g_{i} \subseteq \mathcal{L}_{i}\right\}$. All links together form a network ${ }^{10}$, typically denoted by $g$, so $g \equiv \bigcup_{i \in \mathcal{N}} g_{i}$. The strategy space $\mathcal{G}$ is the set of all possible networks, which is the collection of all subsets of the set of all possible links. Thus $\mathcal{G} \equiv\{g \subseteq \mathcal{N} \times \mathcal{N}: g \subseteq \mathcal{L}\}$. We can depict such a network $g$ in a graph, where the players are the nodes, and each link $i i^{\prime} \in g$ is represented by an arrow (directed arc) from $i$ to $i^{\prime}$. For example, Figure 1 shows the network $\{12,23,43\}$.

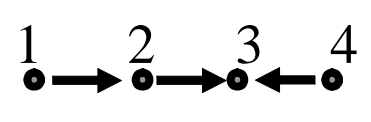

Figure 1: Example of a 4 player network $g$, where $g=\{12,23,43\}$.

Now we come to the (dis)incentives for players to sponsor links. The disincentives arise because sponsoring links is costly. The costs of a link $i j$ are denoted by $c$, and are incurred completely by the sponsor; the recipient incurs no costs. Let $\mathcal{N}_{i}^{S}(g) \subset \mathcal{N}$ be the set of players to whom player $i$ sponsors a link in $g$, so $\mathcal{N}_{i}^{S}(g) \equiv\{j \in \mathcal{N}: i j \in g\}$. Hence the total costs for player $i$ in network $g$ are equal to $\left|\mathcal{N}_{i}^{S}(g)\right| c$.

The benefits of links comes from the need for information by players. Each player owns a unit of (non-rival) private information. Players benefit from accessing the information of other players. A player can access another player's information (only) if the two players are connected by a path of links. On this path, it does not matter who the sponsor of the links are. The benefits of a link

\footnotetext{
${ }^{10}$ Observe that the strategy profile coincides with the network. In this paper we will refer to any strategy profile as a network. Similarly, we will refer to any (strict) Nash equilibrium as a (strict) Nash network.
} 
'flow in two directions'. To make this precise, we let $\overline{i j} \in g$ denote that $i j \in g$ or $j i \in g$ or both ${ }^{11}$. We say that in network $g$ players $i_{0}$ and $i_{k}$ are connected if there exists some subset of players $\mathcal{N}_{i_{0} i_{k}} \subseteq \mathcal{N}, \mathcal{N}_{i_{0} i_{k}}=\left\{i_{0}, \ldots, i_{k}\right\}$ such that for all $\ell \in\{1, \ldots, k\}$ we have that $\overline{i_{\ell-1} i_{\ell}} \in g$. Let $\mathcal{N}_{i}(g)$ denote the set of players to whom player $i$ is connected in network $g$.

When two players are connected, they exchange their private information. However, information decays more the longer the path. We follow the literature (see e.g. Bala and Goyal (2000a)) in our modeling of decay. In this literature only the shortest path between any two players is relevant. The distance between players $i$ and $j$ in network $g$ is the length of the shortest path between these two players. We denote this distance by $d_{i j}(g) .{ }^{12}$ Let $\mathcal{N}_{i}^{d}(g) \subset \mathcal{N}$ be the set of players at distance $d$ from player $i$ in network $g$. So $\mathcal{N}_{i}^{d}(g) \equiv\left\{j \in \mathcal{N}: d_{i j}(g)=d\right\}$, where by definition $\mathcal{N}_{i}^{0}(g)=\{i\}$. Every time the information is passed on a constant fraction $(1-\delta)$ of the (remaining) information is lost, where $\delta \in(0,1)$. The total amount of information gathered by player $i$ in network $g$ is then $I_{i}(g)=\sum_{d=0}^{n-1}\left(\delta^{d}\left|\mathcal{N}_{i}^{d}(g)\right|\right)$. We call $I_{i}(g)$ player $i$ 's ex-post information, as it is the information that $i$ obtains after having decided to sponsor all links in $g_{i}$. The benefits derived by player $i$ from network $g, V_{i}(g)$, are an increasing function of $I_{i}(g)$, specifically $V_{i}(g)=f\left(I_{i}(g)\right)$ where $f^{\prime}>0$.

Observe that decay gives players incentives to sponsor links to players to whom they are already connected for the purpose of reducing the distance between them. However, throughout this paper we assume that $\delta$ is large enough to ensure minimality of any Nash network, meaning that any two players are not connected by more than one path of links. So the amount of decay is small.

The utility which $i$ obtains in $g$ equals his benefits minus his costs. Formally,

$$
U_{i}(g)=V_{i}(g)-\left|\mathcal{N}_{i}^{S}(g)\right| c .
$$

Define $g_{-i}$ as all the links in $g$ excluding the links sponsored by player $i$. A network $g$ is a strict Nash network (SNN) if for each player $i \in \mathrm{N}$ and all $g_{i}^{\prime} \in \mathrm{G}_{i}$, $g_{i}^{\prime} \neq g_{i}$, we have $U_{i}(g)>U_{i}\left(g_{-i} \cup g_{i}^{\prime}\right)$. Similarly, in a Nash network every player plays a best reply strategy. Denote by $B R_{i}^{f}(g)$ the set of best reply strategies of player $i$ versus network $g$ under function $f$. Formally, for any benefit function $f$ we have

$$
B R_{i}^{f}\left(g^{*}\right)=\left\{g_{i} \in \mathcal{G}_{i}: U_{i}\left(g_{-i}^{*} \cup g_{i}\right) \geq U_{i}\left(g_{-i}^{*} \cup g_{i}^{\prime}\right) \text { for all } g_{i}^{\prime} \in \mathcal{G}_{i}\right\} .
$$

If in a Nash network the best reply set for each player is singleton then the network is a SNN. We denote the set of networks that are SNN by $\mathcal{G}^{S N N}$.

\subsection{Terminology and notation}

Having described the game, we introduce some useful terminology and notation. Each network $g$ partitions the population into one or more components

\footnotetext{
${ }^{11}$ So $\overline{i j} \in g$ says that the intersection of $\{i j, j i\}$ and $g$ is not empty.

${ }^{12}$ Note that two-way flow implies $d_{i j}=d_{j i}$ for all $i, j \in N$.
} 
of $g$, where two players belong to the same component if and only if they are connected. Component $x$ is denoted as $C_{x}(g)$. A network that contains only one component is called a connected network. A network is minimal if the deletion of any link in that network will result in an increase of the number of components. A cycle is a set of links $\left\{\overline{j_{0} j_{1}}, \ldots, \overline{j_{k-1} j_{k}}\right\}$ such that $j_{0}=j_{k}$. This implies that a component (or network) is minimal if and only if it contains no cycles. We call a link which is not part of a cycle a minimal link, while any link which is part of a cycle is called a non-minimal link. A non-recipient player is a player that receives no links. ${ }^{13}$ We call a recipient that receives more than one link a multi-recipient. Note that multi-recipients cannot be non-recipient players, since non-recipient players receive no links. E.g, in the network in Figure 1 player 3 is a multi-recipient, players 1 and 4 are both non-recipient players.

We now define our concept of the best-informed player. For a network $g$ and for a set of players $M, M \subseteq \mathcal{N}$, define network $g_{M}$ as the set of links of network $g$ of which both the sponsor and the recipient of the link belong to $M$. Formally, $g_{M}=\{i j \in g: i, j \in M\}$. Note that $g_{M}$ may be, but does not have to be, a component.

Definition 1 Let $M \subseteq \mathcal{N}$ be a connected subset of players in network $g$. Then player $i, i \in M$, is a best-informed player of $M$ if $I_{i}\left(g_{M}\right) \geq I_{j}\left(g_{M}\right)$ for all $j \in M$.

Remark 1 If in network $g$ some player $i$ is not part of component $C_{x}(g)$, then the additional information he receives from sponsoring a link to some player $j, j \in C_{x}(g)$, is $\delta I_{j}(g)$. Since all links cost the same, and because utility is strictly increasing in information the best link which player $i$ can have into the component is to the best-informed player of $C_{x}(g)^{14}$. Moreover, by the same arguments it follows that player $i$ is indifferent between any two best-informed players of a particular component.

Let $i i^{\prime} \in g$, then $A_{i i^{\prime}}(g)$ is the set of players which $i$ observes in $g$ exclusively via link $i i^{\prime 15}$. Using this notation, $g_{A_{i i^{\prime}}(g)}$ is the component to which player $i$ gains access by sponsoring a link to $i^{\prime}$. More generally, $A_{\overline{i i^{\prime}}}(g)$ is the set of players which $i$ observes in $g$ exclusively via link $i i^{\prime}$, where it is left open whether $\overline{i i^{\prime}}=i i^{\prime}$ or $\overline{i i^{\prime}}=i^{\prime} i$. A link $i i^{\prime} \in g$ is said to point to player $j$ if $j \in A_{i i^{\prime}}(g)$. A link $i i^{\prime} \in g$ is said to point away from player $j$ if $j \in A_{\overline{i i^{\prime}}}(g)$ and $\overline{i i^{\prime}}=i^{\prime} i$. Links $i i^{\prime} \in g$ and $j j^{\prime} \in g$ are said to point to each other if $i \in A_{j j^{\prime}}(g)$ and $j \in A_{i i^{\prime}}(g)$. Finally, we say that a link through which the sponsor observes only the recipient of that link is an end link, formally a link $i i^{\prime} \in g$ is an end link if $A_{i i^{\prime}}(g)=\left\{i^{\prime}\right\}$. If $i i^{\prime}$ is an end link then $i$ is an end sponsor and $i^{\prime}$ an end recipient. To illustrate these concepts, we consider network $g$ in Figure 1. It

\footnotetext{
${ }^{13}$ In any connected network, a non-recipient player will also be transmitter, i.e. a player who receives no links while he sponsors at least one link.

${ }^{14}$ Note that without decay (so $\delta=1$ ), every player in any connected set is a most valuable player in that set. This concept can also be useful when considering heterogeneous agents.

${ }^{15}$ So $A_{i i^{\prime}}(g) \bigcap \mathcal{N}_{i}\left(g \backslash i i^{\prime}\right)=\emptyset$ and $A_{i i^{\prime}}(g) \bigcup \mathcal{N}_{i}\left(g \backslash i i^{\prime}\right)=\mathcal{N}_{i}(g)$
} 
consists of one component, and is thus a connected network. In this network, $A_{12}(g)=\{2,3,4\}$, and $A_{23}(g)=\{3,4\} ; g_{A_{12}(g)}=\{23,43\}$ and $g_{A_{23}(g)}=\{43\} ;$ link 23 points to players 3 and 4, and away from players 1 and 2, while links 12 and 43 point towards each other. Finally, there are no end links.

Two networks have the same architecture if one can obtain one network from the other by permuting the strategies of agents. We use $\gamma$ to denote an individual architecture. Two highly stylized architectures that play an important role in our analysis are the center-sponsored star (CSS) and the periphery-sponsored star (PSS). Stars are minimal connected networks where one player, the central player, has a link (either as sponsor or as recipient) with every other player. Networks A-C in Figure 2 are all stars. The CSS is a special case of the star where the central player sponsors each link (Network A), while the PSS s a special case of the star where the central player sponsors each link (Network C). A star with both center-sponsored and periphery-sponsored links (e.g. Network B) is called a hybrid star.

\subsection{Preliminary results}

We now derive some preliminary results. The definitions above imply the following lemma, which relates the number of non-recipient players to the number of multi-recipients in minimal connected networks.

Lemma 1 Let network $g$ be a non-empty minimal connected SNN. Then $g$ either contains one or more multi-recipients, in which case it contains multiple non-recipient players; in this case, we call g a multi-recipient network. Or $g$ contains no multi-recipients, in which case it contains exactly one non-recipient player; in this case, we call $g$ a non-recipient player network.

Proof. Any minimal connected directed graph has precisely $(n-1)$ directed links. These can be received by at most $(n-1)$ different recipients. It follows that any minimal connected network contains at least one non-recipient player. If there are no multi-recipients, then the directed links are each received by a different player, so that there is exactly one non-recipient player. If there is at least one multi-recipient, at least two of the $(n-1)$ directed links are received by a multi-recipient, meaning that at least two players will not receive any links. It follows that there must be at least two non-recipient players.

Further, we show that there exists a SNN, and that for small decay, all nonempty SNNs are minimal and connected. We first show that in the absence of decay, non-empty SNNs are always minimal.

Lemma 2 In the absence of decay, no player in any network $g$ prefers to sponsor non-minimal links.

Proof. Any non-minimal link yields a marginal benefit of zero. As linking is costly, a player sponsoring a non-minimal link is better off when deleting any one of his non-minimal links. 
We now define two levels threshold levels of decay, and after that we show that both are smaller than one.

Definition 2 Let $\delta_{M}(c, n, f(I))$ be the lowest level of decay such that for all $\delta>\delta_{M}(c, n, f(I))$ no player in any network prefers to sponsor a non-minimal link.

Definition 3 Let $\delta_{R}(c, n, f(I))$ be the lowest level of decay such that for all $\delta>\delta_{R}(c, n, f(I))$ any $S N N g$ is minimal.

We now show that both thresholds are strictly below 1 .

Lemma 3 For any $c>0, n \geq 3$, and $f(I)>0$ we have $\delta_{R}(c, n, f(I)) \leq$ $\delta_{M}(c, n, f(I))<1$.

Proof. Note that the benefit function is continuous in $\delta$. By this continuity it follows from Lemma 2 that $\delta_{M}(c, n, f(I))<1$. From the two definitions it is apparent that $\delta_{R}(c, n, f(I)) \leq \delta_{M}(c, n, f(I))$, since if all players prefer to delete non-minimal links, no SNN can contain a cycle.

The condition $\delta>\delta_{M}(c, n, f(I))$ ensures that, in any given network, no player wants to add a non-minimal link. Yet, even if this condition is not valid, it may still be that all SNN are minimal. If there is a network to which a player prefers to add a non-minimal link, then this new network itself need not be a SNN. This is why the condition $\delta>\delta_{R}(c, n, f(I))$, ensuring that all SNN are minimal, cannot be a stronger condition than $\delta>\delta_{M}(c, n, f(I))$, as stated in Lemma 3.

The following two lemmas show together that all non-empty networks are connected ${ }^{16}$.

Lemma 4 Let network $g$ be a non-empty $S N N$ and $\delta>\delta_{R}(c, n, f(I))$. Then $g$ has no singleton component.

Proof. Suppose not. Then there exists a player, say $j$, who is isolated. Moreover, by minimality and non-emptiness of $g$, there exists some player $i$ who receives no links, but does sponsor a set of links himself, namely to all players in $\mathcal{N}_{i}^{S}(g)$. Note that $I_{i}\left(g_{-i}\right)=I_{j}\left(g_{-i}\right)=I_{j}(g)$. Now let player $j$ consider strategy $g_{j}^{\prime}$ in which he sponsors a link to every recipient of links from player $i$. So $g_{j}^{\prime}=\left(j i^{\prime} \in \mathcal{L}: i^{\prime} \in \mathcal{N}_{i}^{S}(g)\right)$. This costs him the same as $g_{i}$ costs player $i$. But the benefits to player $j$ are strictly larger because he accesses the same players at the same distance as player $i$ does and in addition he will be connected to player $i$. Hence if $g_{i}$ is a best reply for player $i$ to network $g$, then $g_{j}=\{\emptyset\}$ cannot be a best reply to player $j$. Since $g$ is a SNN by assumption, this forms a contradiction.

\footnotetext{
${ }^{16}$ This result is a variant of Bala and Goyal (2000a), Proposition 5.1, which applied to linear benefit functions only.
} 
Lemma 5 Let network $g$ be a non-empty $S N N$ and $\delta>\delta_{R}(c, n, f(I))$. Then $g$ is connected.

Proof. Suppose not. Then by Lemma 4 there is a strict Nash network $g$ which contains multiple non-singleton components. Without loss of generality we have $i i^{\prime}, j j^{\prime} \in g$ such that $i$ and $i^{\prime}$ belong to one component, say $C_{1}(g)$, and $j$ and $j^{\prime}$ to another, say $C_{2}(g)$. Because $g$ is a strict Nash network, player $i$ prefers to sponsor a link to $i^{\prime}$ and not to any player in $C_{2}(g)$. So player $i^{\prime}$ has access to more information in $g \backslash\left\{i i^{\prime}\right\}$ than $j^{\prime}$ in $g$. Hence we obtain that $I_{i^{\prime}}(g)>I_{i^{\prime}}\left(g \backslash\left\{i i^{\prime}\right\}\right)>I_{j^{\prime}}(g)>I_{j^{\prime}}\left(g \backslash\left\{j j^{\prime}\right\}\right)$. Because $g$ is Nash, we also have that $I_{i^{\prime}}(g)<I_{j^{\prime}}\left(g \backslash\left\{j j^{\prime}\right\}\right)$, which gives us a contradiction. Hence any Nash network has only one component and is therefore connected.

So all non-empty SNNs are connected. Naturally, if costs are low enough the empty network is not a SNN, implying that all SNNs are connected. Since the minimal benefit an isolated player would derive from sponsoring a link is $f(1+\delta)-f(1)$, Lemma 5 implies the following corollary.

Corollary 1 Let $c<f(1+\delta)-f(1)$, then any network $g$ which is a $S N N$ is connected.

Bala and Goyal (2000a) pointed out that there always exists a strict Nash network. For example, for $c \geq f\left(1+\delta+(n-2) \delta^{2}\right)-f(1)$ the empty network is a SNN, while for $c<f\left(1+\delta+(n-2) \delta^{2}\right)-f(1)$ the PSS is a SNN.

Before starting our actual characterization, we have thus shown that for small decay all non-empty SNN must be minimal connected networks.

\section{Characterization}

The preliminary results of the previous section show that for small decay, all non-empty SNNs are minimal and connected. In this section we will provide a partial characterization of all minimal connected SNNs.

Specifically, we characterize the necessary structural properties for any minimal connected SNNs. This also covers minimal connected SNNs when there is sufficient decay to allow for some non-minimal SNNs. It follows that all minimal connected SNNs satisfy these properties for small decay (here for all $\left.\delta>\delta_{R}(c, n, f(I))\right)$. In Section 5 we will combine this with an additional property, the balancing condition, to complete the characterization of non-empty SNNs under small enough decay, as the balancing condition allows us to supply sufficient structural properties ${ }^{17}$.

The characterization in this section consists of two parts, namely Propositions 1 and 2. Proposition 1 presents structural properties that every minimal

\footnotetext{
${ }^{17}$ This will require an additional upper bound on the degree of decay. Therefore the results in this section and Section 4 may hold for a range of decay which is not considered in Section 5. For this reason we present the results without the balancing condition first, and then add the balancing condition to the characterization.
} 
connected SNN must have. Proposition 2 and its Corollary 3 relate the structural position of a player in the network to the player's informedness. Both propositions build further on the following lemma, which roughly states that links in a SNN are sponsored to the recipients who have most information among the set of players accessed through the link.

Lemma 6 Let $g$ be a minimal connected $S N N$. For all ij $\in g$, it must be the case that, among the players in the set $A_{i j}(g), j$ is the unique best-informed player in network $g_{A_{i j}(g)}$, and among the players in the set $A_{i j}(g)$ is the one to receive strictly the most information from $g$.

Proof. Consider any minimal network $g^{\prime}$, and any sponsor of a link, say player $i$ with link $i j \in g^{\prime}$. Then the amount of information gained by $i$ through $i j \in g^{\prime}$ equals $\delta I_{j}\left(g_{A_{i j}\left(g^{\prime}\right)}^{\prime}\right)$, namely the amount of information which $j$ gathers in the network without link $i j$, discounted one time. If $i$ would replace $i j$ by the link $i j^{\prime}$, where $j^{\prime} \in A_{i j}\left(g^{\prime}\right)$, then $i$ would receive $\delta I_{j^{\prime}}\left(g_{A_{i j}\left(g^{\prime}\right)}^{\prime}\right)$ instead. As each link costs the same $i$ strictly prefers $i j$ to $i j^{\prime}$ if and only if $I_{j}\left(g_{A_{i j}\left(g^{\prime}\right)}^{\prime}\right)>$ $I_{j^{\prime}}\left(g_{A_{i j}\left(g^{\prime}\right)}^{\prime}\right)$. As network $g$ is a SNN, $i j \in g$ thus implies that $I_{j}\left(g_{A_{i j}(g)}\right)>$ $I_{j^{\prime}}\left(g_{A_{i j}(g)}\right)$ for any $j^{\prime} \in A_{i j}(g)$. Moreover, $j$ is also the best-informed player of $A_{i j}(g)$ in network $g$. The reason is that the link $i j$ increases the information advantage of player $j$ over the other players in $A_{i j}(g)$, since the information which travels from $i$ to $j$ will decay at least one more time before it reaches any other player in $A_{i j}(g)$. This concludes the proof.

Lemma 6 allows us to derive a result central to the characterization, namely that links pointing to one another must be sponsored to the same player.

Lemma 7 Let $g$ be a minimal connected $S N N$. If $j \in A_{i i^{\prime}}(g)$ and $i \in A_{j j^{\prime}}(g)$ (meaning that links $i i^{\prime}$ and $j j^{\prime}$ point towards one another), then $i^{\prime}=j^{\prime}$.

Proof. We prove this by contradiction. Suppose not, so $i^{\prime} \neq j^{\prime}$. Note that by minimality there is one path connecting $i$ and $j$, and this path goes via players $i^{\prime}$ and $j^{\prime}$. By Lemma 6, among the nodes in the set $A_{i i^{\prime}}(g), i^{\prime}$ is the node that receives strictly the most information from network $g$, and in the set $A_{j j^{\prime}}(g), j^{\prime}$ is the node that receives strictly the most information from network g. But as $j^{\prime} \in A_{i i^{\prime}}(g)$ and $i^{\prime} \in A_{j j^{\prime}}(g)$ we have that both $I_{i^{\prime}}(g)>I_{j^{\prime}}(g)$ and $I_{j^{\prime}}(g)>I_{i^{\prime}}(g)$ : a contradiction.

This implies that if no end-link is sponsored in a minimal connected SNN, then all links are received by the same player. As this insight proves useful in Section 4, we state it in the following Corollary.

Corollary 2 Let $g$ be a minimal connected SNN. Then $g$ is either a PSS, or $g$ contains at least one end link. 
From Lemma 7, we can derive a set of necessary conditions which all minimal connected SNNs satisfy.

Proposition 1 Let $g$ be a minimal connected SNN. Then $g$ has the following properties:

1. There is a unique characterizing player in g. If a multi-recipient player exists in $g$, then he is the unique multi-recipient in $g$, and he will be the characterizing player of $g$. If no multi-recipient player exists in $g$, then there exists a unique non-recipient player in $g$ and he will be the characterizing player of $g$.

2. Network links tend to be outward-oriented in the following way:

(a) if the characterizing player is a multi-recipient, all links point away from him, except for those links of which the characterizing player is the recipient.

(b) if the characterizing player is a non-recipient player, then all links point away from him.

3. Every recipient of a link in a SNN either has no other links, or at least two other links.

Proof. ad 1. We know from Lemma 1 that every minimal connected graph either has one or more multi-recipients, or has no multi-recipients, in which case the minimal connected graph has a single non-recipient player. It remains to be shown that networks with one or more multi-recipients in fact can only have a single multi-recipient. Suppose that players $i$ and $j$ are both multi-recipient players. Because $g$ is minimally connected, players $i$ and $j$ are connected by at most one path. This means that both players receive at least one link which is not part of the path connecting them. These links point toward each other which implies by Lemma 7 that $i=j$. Since $i$ and $j$ were arbitrarily chosen multirecipient players, it follows that there cannot be more than one multi-recipient player.

ad 2a. Let player $i^{\prime}$ be a multi-recipient player and let there be any link pointing towards $i^{\prime}$. So there exists some $j j^{\prime} \in g$ such that $i^{\prime} \in A_{j j^{\prime}}(g)$. Since player $i^{\prime}$ receives at least two links, there exists some player $i \in A_{j j^{\prime}}(g)$ such that $i i^{\prime} \in g$. It follows that $j \in A_{i i^{\prime}}(g)$. By Lemma 7 it must be that $j^{\prime}=i^{\prime}$.

ad 2 b. Let $i$ be the non-recipient characterizing player. This implies that there are no multi-recipient players in the network. We will now show that if property $2 \mathrm{~b}$ is not satisfied, the network does contain a multi-recipient player: a contradiction. Suppose that there exists a link $j j^{\prime} \in g$ such that $i \in A_{j j^{\prime}}(g)$. Because $g$ is minimally connected and because $i$ receives no links, there exists a link $i i^{\prime} \in g$ such that $j^{\prime} \in A_{i i^{\prime}}(g)$. Lemma 7 then implies that $i^{\prime}=j^{\prime}$, which in turn implies that player $i^{\prime}$ receives at least two links, namely one from $i$ and one from $j$. Thus $i^{\prime}$ is a multi-recipient player, which cannot be. 
ad 3. Suppose not. Then $i j \in g$, and $j$ has a single link $\overline{j k}$. There are two cases: either $A_{i j}(g)$ contains players other than $j$ and $k$, or not. If not, $i$ would be indifferent between the link $i j$ and $i k$ since either link would give him access to one player at distance 1 and one at distance 2 . This cannot be the case, since $g$ is a SNN. Thus other players are observed as well $\left(A_{i j}(g) \backslash\{j, k\}\right.$ is not empty). However, then $i$ strictly prefers to replace $i j$ by $i k$, since replacing the link would bring all players in $A_{i j}(g) \backslash\{j, k\}$ one step closer as well: a contradiction.

We will now relate the characterizing player identified in Proposition 1 to the best-informed player in the network. We show that, if the characterizing player is a multi-recipient player, he is the unique best-informed player. If the characterizing player is a non-recipient player, either he is the best-informed player, or one of the recipients of the links he sponsors. When centrality is measured in terms of information gathered (more central players acquire more information), it appears that the characterizing player who is a multi-recipient player is actually the central player of the network as well. In the same way, a non-recipient characterizing player either is himself a central player, or he sponsors a link to each central player in the network. In that sense the characterizing player is always central or almost central in the network.

Proposition 2 Let $g$ be a minimal connected SNN. Then,

1. if the characterizing player is a multi-recipient player, then he is the unique best-informed player in $g$.

2. if the characterizing player is a non-recipient player, then the best-informed player is either the non-recipient player himself, or one of the recipients of links sponsored by the non-recipient player.

Proof. Let player $i_{V}$ be the best-informed player and $i_{c h}$ the characterizing player in $g$. We show Part 1 by contradiction. If $i_{c h}$ is not the best-informed player in $g$, i.e. $i_{c h} \neq i_{V}$, then because $i_{c h}$ receives multiple links, there exists at least one player $i$ sponsoring a link to $i_{c h}$ such that $i_{V} \in A_{i i_{c h}}(g)$. By Lemma $6, i_{c h}$ is the player receiving most information from $g$ among the set $A_{i i_{c h}}(g)$. But this contradicts $i_{V}$ being the best-informed player.

Part 2: there are two possibilities. Either $i_{c h}$ is the best-informed player, or $i_{c h}$ is not the best-informed player. The first case satisfies our claim. In the second case, by connectedness (Lemma 5) $i_{c h}$ sponsors a link, say $i_{c h} j \in g$, through which he observes $i_{V}$. By Lemma $6, j$ is the player receiving most information from $g$ among the set $A_{i_{c h} j}(g)$, so that $i_{V}=j$.

Further, as a direct Corollary of Lemma 6, we can show that more peripheral players tend to obtain less information in any SNN.

Corollary 3 Let $g$ be a minimal connected SNN. Consider any path of links in $g$ pointing in the same direction, $\left\{j_{0} j_{1}, j_{1} j_{2}, \ldots, j_{k-1} j_{k}\right\}$. Then $I_{j_{1}}(g)>I_{j_{2}}(g)>$ $\ldots>I_{j_{k-1}}(g)>I_{j_{k}}(g)$. 


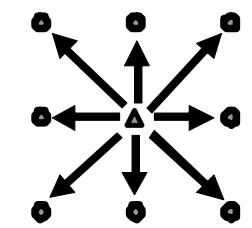

(A)

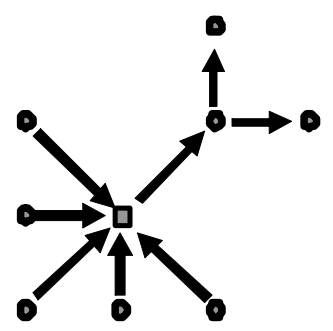

(D)

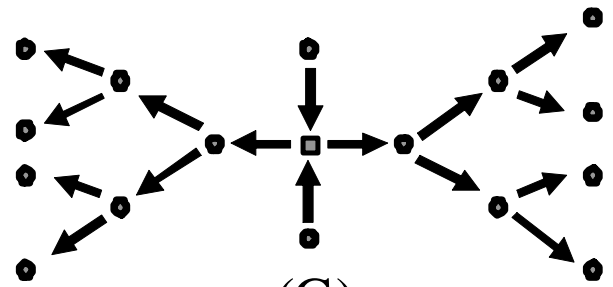

(G)

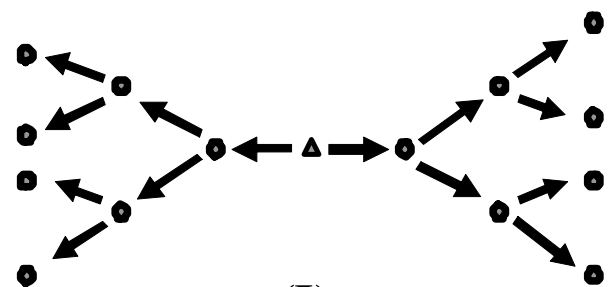

(I)

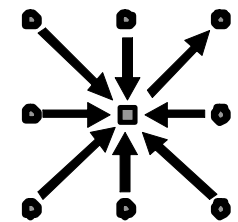

(B)

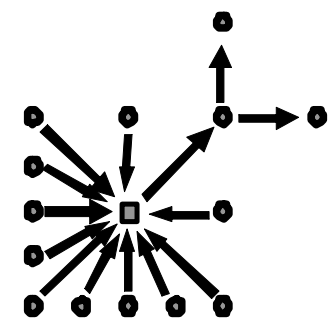

(E)

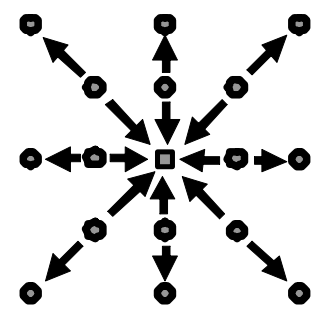

(F)

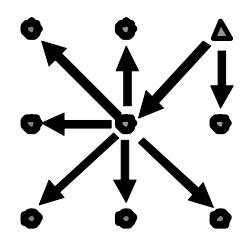

(H)
(C)

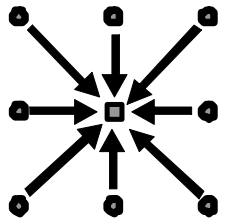


Proof. By Lemma 6 , among all players in the set $A_{j_{0} j_{1}}(g)$, player $j_{1}$ obtains the most information from $g$. Further, among all the players in $A_{j_{1} j_{2}}(g) \subset A_{j_{0} j_{1}}(g)$, player $j_{2}$ obtains the most information from $g$. The rest of the proof follows by induction.

All minimal connected networks which satisfy the characterization of Proposition 1 are called candidate networks. We denote the set of these networks as $\mathcal{G}^{C A N}$. Denoting by $\mathcal{G}^{M I N C O N}$ the set of all minimal connected networks, we then know that for small decay $\left(\delta>\delta_{R}(c, n, f(I))\right) \mathcal{G}^{C A N} \subset \mathcal{G}^{M I N C O N}$, as Proposition 1 clearly restricts the set of candidate SNN. Further, denoting by $\mathcal{G}^{S N N}$ the set of SNN and by $g^{e}$ the empty network, it is clear that $\mathcal{G}^{S N N} \backslash g^{e} \subset \mathcal{G}^{C A N}$, i.e. the set $\mathcal{G}^{C A N}$ does not characterize the set of nonempty SNN. This is for two reasons.

First, while the necessary conditions in Proposition 1 are based on the result that each sponsor should sponsor a link to the unique best-informed player in the accessed component (Lemma 6), we did not provide a rule specifying how the best-informed player in a given component is identified. Indeed, in some of the networks of Figure 2, the identity of the best-informed player in any accessed component is ambiguous, as it depends on the level of decay. But it depends on nothing else. For this reason, we denote by $\mathcal{G}^{C A N}(\delta)$ the set of candidate networks which satisfy Lemma 6 for a given level of $\delta$. Given the ambiguity in identifying the best-informed player, it follows that $\mathcal{G}^{C A N}(\delta) \subset \mathcal{G}^{C A N}$.

Second, $\mathcal{G}^{C A N}$, and for that matter $\mathcal{G}^{C A N}(\delta)$, does not characterize the set of non-empty SNNs because in the networks in $\mathcal{G}^{C A N}$ and $\mathcal{G}^{C A N}(\delta)$, some players may still have incentives to delete links, because the cost $c$ of sponsoring links make one or more links too expensive. It follows that $\mathcal{G}^{S N N} \backslash g^{e} \subseteq \mathcal{G}^{C A N}(\delta)$. Summarizing, we thus have $\mathcal{G}^{S N N} \backslash g^{e} \subseteq \mathcal{G}^{C A N}(\delta) \subset \mathcal{G}^{C A N} \subset \mathcal{G}^{M I N C O N}$.

In the rest of the paper, we characterize $\mathcal{G}^{S N N} \backslash g^{e}$ as tightly as possible, for a set of parameters as general as possible. In Section 4, we look first at the condition that in an SNN, no player should want to delete a link. We can investigate this question for general $\mathcal{G}^{C A N}(\delta)$, without actually characterizing a rule identifying the best-informed player in any component as a function of $\delta$. In Section 5, we characterize $\mathcal{G}^{C A N}(\delta)$ for high $\delta$. We do this by showing that for high $\delta$, networks where each sponsor accesses a unique best-informed player in any accessed component, should meet a condition which we call the balancing condition. We then show that any balanced network, namely a minimal connected network which satisfies the balancing condition and the conditions of Proposition 1, is a SNN for some positive range of parameters. Thus we fully characterize $\mathcal{G}^{S N N} \backslash g^{e}$ for a particular range of parameters. Before doing this, however, in the next section we first derive our results about the relative stability of network in $\mathcal{G}^{C A N}(\delta)$, which are valid for a weakly wider range for $\delta$ than the range implied by the balancing condition. 


\section{Marginal benefits of information and relative stability}

In this section we compare the relative stability of the different network architectures in $\mathcal{G}^{C A N}(\delta)$, i.e. of candidate networks where for a specific level of $\delta$, it is the case that Lemma 6 is valid. We restrict our attention to $\delta>\delta_{M}(c, n, f(I)) .{ }^{18}$ By $\delta>\delta_{M}(c, n, f(I))$ we know that for any $g \in \mathcal{G}^{C A N}(\delta)$ no player wishes to add a link to the already connected network $g$. Moreover, by Lemma 6 and the construction of $\mathcal{G}^{C A N}(\delta)$ we know that no player wishes to replace a link. Hence, for the given parameters, we only need to consider whether players want to delete one or more of their links.

To be precise, we say that, for a given decay factor $\delta$, a candidate architecture $\gamma_{1}$ is weakly more stable than another candidate architecture $\gamma_{2}$ if stability of $\gamma_{2}$ implies stability of $\gamma_{1}$. We say that $\gamma_{1}$ is strictly more stable than $\gamma_{2}$ if stability of $\gamma_{2}$ implies stability of $\gamma_{1}$ but not the other way around. We say that a candidate network is strictly (weakly) the most stable if it is strictly (weakly) more stable than any other candidate architecture. Finally, we say that two architectures $\gamma_{1}$ and $\gamma_{2}$ are equally stable if stability of $\gamma_{1}$ implies stability of $\gamma_{2}$, and vice versa.

Whether a player wishes to delete a link he sponsors depends on two factors. First, this depends on the amount of additional information he gains by this link. Second, it depends on how much he is willing to pay for the link. The second factor in turn depends on the ex-ante information of the player. We consider three specific benefit functions. Under CMBI, the ex-ante information of the player is irrelevant, as his willingness to pay for a link only depends on the amount of information he receives. Under DMBI, the less ex-ante information the player has, the more he is willing to pay for additional information. Finally under IMBI, players are willing to pay more for additional information when they have more ex-ante information already. ${ }^{19}$ We come to the following result for these benefit functions.

Proposition 3 For any $n$ and $f(I)$, fix any $\delta$ and $c$ such that $\delta>\delta_{M}(c, n, f(I))$ and such that $\mathcal{G}^{S N N}$ contains a non-empty network. Then for the n-player networks in $\mathcal{G}^{C A N}(\delta)$

1. under CMBI, the PSS is strictly the most stable architecture, and all nonPSS architectures are equally stable.

\footnotetext{
${ }^{18}$ Note that $\delta_{M}(c, n, f(I))$ is decreasing in $c$ : the higher the costs, the less willing players are to sponsor non-minimal links.

${ }^{19}$ The network formation literature often focuses on CMBI benefit functions. For instance, Bala and Goyal (2000a) consider only CMBI when they discuss decay. DMBI is a natural result if all players get a private signal from a common distribution. The first signals are more informative than later signals. IMBI is a natural assumption (at least until some information level), when some pieces of information are hard to interpret without other pieces of information. In for instance Cohen and Levinthal (1989) knowledge of old research results increases how much the agent can learn from new research results.
} 
2. under DMBI, the PSS is strictly the most stable architecture. All CSS and hybrid star architectures are equally stable, and are strictly less stable than any of the other network architectures.

3. under IMBI, a star with $k$ center-sponsored links is weakly more stable than stars with $k^{\prime}$ center-sponsored links if $k^{\prime}>k$. Moreover for any nonstar network $\hat{g}$ there exists a hybrid star, $g^{*}$, which is weakly more stable if $\hat{g}$ has diameter 3 or 4 as well as a non-recipient characterizing player, and which is strictly more stable otherwise.

Proof. Consider any network $g \in \mathcal{G}^{C A N}(\delta)$ and see whether it is a SNN. First note that by Lemma 6 and the construction of $\mathcal{G}^{C A N}(\delta)$ no sponsor wants to replace his link. Second, by $\delta>\delta_{M}(c, n, f(I))$ no player prefers to sponsor an additional link. Hence if a network is not a SNN, then it must be that some player prefers to delete a link. In the remainder of the proof we will compare across networks the players who are in each network least willing to sponsor their links.

Ad 1. Under CMBI, the only concern of the player is the amount of information he gains through the link. Consider any link in a PSS architecture: each sponsor gains $\left[\delta+(n-2) \delta^{2}\right]$ information through his link. This is the maximal amount of information which can be gained from a single link. In contrast, by Corollary 2 each non-PSS architecture in $\mathcal{G}^{C A N}$ contains an end sponsor. This sponsor gains only $\delta$ by that link, which is the minimal amount of information which can be gained through a link in a minimal network. Hence the willingness for end links is lower than that for the links in the PSS. Hence the PSS is strictly most stable, while all other networks are equally stable.

Ad 2. Under DMBI the amount of ex-ante information is also relevant: the less ex-ante information the more the sponsor is willing to sponsor his links. Now note that any sponsor in the PSS has the minimal possible ex-ante information, namely 1 . Combined with the maximal amount of additional information gained by these sponsors (see ad 1.) the PSS is strictly the most stable network. Consider now the other stars. The central player in such stars, sponsors a link through which he receives minimal extra information $(\delta)$, while he has maximal ex-ante information (specifically: $1+(n-2) \delta$ ). Hence under DMBI any star with at least one center-sponsored link is a least stable network.

Finally consider any other network $g$ in $\mathrm{G}^{C A N}(\delta)$. So $g$ is not a star. All sponsors in $g$ gain less information by their links than the sponsors in the PSS, while they have at least as much ex-ante information. Similarly all sponsors in $g$ gain at least the same amount of additional information as the central player in a hybrid star or CSS does. However, each of these players has strictly less ex-ante information. Hence all those sponsors are willing to pay more for their link than a central player in a star for any one of his links. Concluding, the PSS architecture is strictly most stable, all other stars are strictly least stable, and any other $g \in \mathcal{G}^{C A N}(\delta)$ are strictly in between in terms of stability.

Ad 3.

(i) We will first show that if the PSS is not stable, than each non-recipient player (weakly) prefers to delete all links. Suppose that the PSS is not stable. 
Then, by the fact that $\delta>\delta_{M}(c, n, f(I))$, this must be because sponsoring no links and receiving 1 unit of information is (weakly) better than sponsoring $k$ links and receiving $\left(1+k \delta+(n-1-k) \delta^{2}\right)$ information in total. As a non-recipient sponsoring $k$ links cannot gain more information than $\left(1+k \delta+(n-1-k) \delta^{2}\right)$ (namely $k$ players at distance 1 and the rest at distance 2), it follows that if the PSS is not stable, no network with a non-recipient sponsor is stable. Thus the PSS is weakly most stable among all networks in $\mathcal{G}^{C A N}(\delta)$, as $\mathcal{G}^{C A N}(\delta) \subset$ $\mathcal{G}^{M I N C O N}$ and as all minimal connected networks have at least one non-recipient sponsor $^{20}$.

(ii) We now derive the result that the stability of the star is non-decreasing in the number of center-sponsored links. Suppose that the peripheral-sponsored links are stable. First observe that each link a central player in a star sponsors gives him $\delta$ additional information. Combined with the IMBI benefit function, it follows that if a central player would prefer to delete one link, then he would prefer to delete all his other links too. The central player prefers to keep all his links if the average benefit of those links outweighs the average costs $(c)$. Consider the central players of two alternative stars, where the second star has more center-sponsored links than the first. Let $i_{c}$ be the central player of the first star, where $i_{c}$ sponsor $k$ links. Let $i_{c}^{\prime}$ be the central player of the second star, where $i_{c}^{\prime}$ sponsor $k^{\prime}$ links, thus $k^{\prime}>k$. Notice that the average benefits which $i_{c}^{\prime}$ receives on his 'last' $k$ links is the same as the average benefit which $i_{c}$ receives for his last $k$ links, namely $\frac{1}{k}[f(1+(n-1) \delta)-f(1+(n-1-k) \delta)]$. However $i_{c}^{\prime}$ has a lower average benefit over his 'first' $k^{\prime}-k$ links, as his ex-ante information is lower when he has less links. Thus $i_{c}^{\prime}$ has a lower average benefit of his $k^{\prime}$ links than $i_{c}$ of his $k$ links. Hence the central player is less willing to sponsor his links the more links he has to sponsor. Thus stars are weakly more stable the less links are center-sponsored (note that if the sponsor most willing to delete his link is a peripheral sponsor, any two stars with peripheral sponsors are equally stable).

(iii) Now we show that for any non-star network $\hat{g}, \hat{g} \in \mathcal{G}^{C A N}(\delta)$, there is a hybrid star $g^{*}$ which is weakly more stable. First we construct $g^{*}$. By Corollary $2 \hat{g}$ contains an end sponsor, say $j$. Let $j$ sponsor $k$ end links. Then let $g^{*}$ be a star network where the central player, $j^{*}$, sponsors $k$ links. Second we show that $j$ in network $\hat{g}$ has strictly more incentives incentives to delete his links than $j^{*}$ in $g^{*}$. The $k$ least informative links of $j$ in $\hat{g}$ are equally informative as the $k$ links by $j^{*}$ in $g^{*}$ (specifically $\delta$ information per link). However $I_{j^{*}}\left(g^{*}\right)>I_{j}(\hat{g})$, so $j$ is strictly more willing to delete his $k$ end links in $\hat{g}$ than $j^{*}$ is in $g^{*}$. Thus if $\hat{g}$ is weakly more stable than $g^{*}$, then it is a peripheral sponsor in $g^{*}$, say $i^{*}$, and not central player $j^{*}$ who wants to delete his links in $g^{*}$. Note however, that if $g^{*}$ is unstable because $i^{*}$ prefers to delete his link, then neither is the PSS stable. As the PSS is the weakly most stable architecture, this implies that $\hat{g}$ is unstable too. Thus $g^{*}$ is weakly more stable than $\hat{g}$.

(iv) Finally suppose that $\hat{g}$ and $g^{*}$ are equally stable. So if $g^{*}$ is not stable,

\footnotetext{
${ }^{20} \mathrm{By}$ minimality, there is at least one non-recipient. By connectedness this recipient sponsors a link.
} 
it is not the central player who prefers to delete his links. Let $i^{*}$ be a peripheral sponsor in star $g^{*}$. Suppose that $i^{*}$ is indifferent between keeping and deleting his link. Then

- the PSS is not a SNN as the peripheral players in the PSS are indifferent too $\left(\right.$ as $\left.f\left(1+\delta+(n-2) \delta^{2}\right)-f(1)=c\right)$.

- $\hat{g}_{i}$ is a best reply for each player in $\hat{g}$. If not, then $\hat{g}$ is strictly less stable then $g^{*}$.

- each non-recipient player $i$ in $\hat{g}$, weakly prefers to delete all their links. This follows directly from ad 3.(i) above.

- in network $\hat{g}$ no non-recipient player, say player $i$, is at more than distance 2 from any other player. Suppose not and let, without loss of generality $i$ sponsor $k$ links in $\hat{g}$. Then $i$ is indifferent between $f(1)$ and $f\left(I_{i}(\hat{g})\right)-$ $k c$, where $f\left(I_{i}(\hat{g})\right)<f\left(1+k \delta+(n-k-1) \delta^{2}\right)$, as some information has to travel at least distance three. However, this would imply that $f\left(1+k \delta+(n-k-1) \delta^{2}\right)-k c>f(1)$, which is impossible (see ad 3.(i)).

- $\hat{g}$ has a unique non-recipient player. Suppose not, and let $i$ and $i^{\prime}$ be nonrecipient players. As neither $i$ nor $i^{\prime}$ receives a link they are connected because they both sponsor a link to a third player, say $i^{\prime \prime}$. By the previous bullet, $i^{\prime \prime}$ cannot be at a distance of 2 or more, because then either $i$ or $i^{\prime}$ would be at distance 3 or more of that player. This implies that $i^{\prime \prime}$ is the center of a star. As $\hat{g}$ is a non-star network, this is a contradiction.

Thus if $\hat{g}$ is equally stable as $g^{*}$, then $\hat{g}$ is equally stable as the PSS (first bullet). Moreover, $\hat{g}$ has a unique non-recipient player (last bullet). Thus there is no multi-recipient player, and the non-recipient player is the characterizing player. Finally, the diameter of $\hat{g}$ is maximally 4 (fourth bullet). This concludes the proof.

With respect to the case of IMBI we want to remark the following.

Remark 2 Consider the case of IMBI, and suppose that $\hat{g}$ and $g^{*}$ as defined in Proposition 3, point 3, are equally stable. Let $i$ be the non-recipient player of $\hat{g}$, where $i$ sponsors $l$ links. Then equal stability of $\hat{g}$ and $g^{*}$ implies that

$$
f\left(1+\delta+(n-2) \delta^{2}\right)-c=I_{i}(\hat{g})-l c .
$$

If $l=1$, then because $\hat{g}$ is not a star, $I_{i}(\hat{g})<f\left(1+\delta+(n-2) \delta^{2}\right)$. Thus, if the equality above holds, $l$ is at least two. Moreover, if $l$ is at least two, the equality will only hold for very specific combinations of $\delta$ and c. Hence, it is almost always the case that $\hat{g}$ is strictly less stable than $g^{*}$, even if $\hat{g}$ has a non-recipient characterizing player and a diameter of 4 or less. 
Among the set of minimal connected networks, stars are obviously efficient networks: they keep everyone as close as possible. As we have seen in Section 3 , our characterization does not exclude inefficient, large-diameter networks. However, it remains of interest which networks are SNN under the widest range of linking costs, including large linking costs. For CMBI, the PSS is strictly the most stable network; all other candidate networks are less stable than the PSS, but equally stable with respect to one another. For IMBI, stars in general, whether PSS or not, tend to be more stable, in that for any non-star network there exists a hybrid star network that is not a PSS and that is (almost always) strictly more stable. For DMBI, however, as shown in Proposition 3, non-PSS stars are less stable than candidate larger-diameter networks. Lemma 8 now shows that parameters exist such that there is no SNN of diameter 3, but there is a SNN of diameter 4. This Lemma can the immediately be used to show in Proposition 4 that under DMBI, for large $c$ a gap exists in the diameters covered by the networking in $\mathcal{G}^{S N N}$.

Lemma 8 Let $n \geq 7$ and fix $\delta$ and $c$ such that $\delta>\delta_{M}(c, n, f(I))$. Then under $D M B I$ a candidate network $g$ of diameter 4, say network $g^{4} \in \mathcal{G}^{C A N}(\delta)$, is strictly more stable than the most stable candidate network of diameter 3 .

Proof. For $n \geq 7$, we can construct a diameter 4 candidate network which is strictly more stable than any diameter 3 network. Take the PSS and delete the links sponsored by two distinct peripheral players, $j$ and $j^{\prime}$. Consider two distinct other peripheral players, $i$ and $i^{\prime}$ and let them form the links $i j$ and $i^{\prime} j^{\prime}$. This network, say $g^{4}$, has diameter 4 . The added benefit of $i j$ (and thus of $\left.i^{\prime} j^{\prime}\right)$ is $f\left(1+2 \delta+(n-4) \delta^{2}+\delta^{3}\right)-f\left(1+\delta+(n-4) \delta^{2}+\delta^{3}\right)$. The added benefit of each other link in $g^{4}$ is higher. Moreover each link is sponsored to the best-informed player of the accessed set of players and by $\delta>\delta_{M}(c, n, f(I))$ no player prefers to add a link. Thus if $g^{4}$ is not a SNN, then it must be that the added benefit of $i j$ does not outweigh its $\operatorname{costs} c$. Moreover, if any link is unstable, then $i j$ and $i j^{\prime}$ are unstable too.

Now we construct the most stable network of diameter 3 . In all diameter 3 networks we can uniquely identify two adjacent players, say $i_{c}$ and $i_{c}^{\prime}$ such that for any other player $j$ we have that either $\overline{i_{c} j} \in g$ or $\overline{i_{c}^{\prime} j} \in g$. Of the remaining $(n-2)$ players let $k$ players, with $1 \leq k \leq(n-3)$, have a link (as sponsor or recipient) with $i_{c}$ and $(n-k-2)$ have a link $i_{c}^{\prime}$. By Lemma 7 some end link is sponsored by either $i_{c}$ or $i_{c}^{\prime}$. Suppose, without loss of generality, that $i_{c}$ sponsors an end link. In that case, this end link gives him an added benefit of $f\left(1+(k+1) \delta+(n-k-2) \delta^{2}\right)-f\left(1+k \delta+(n-k-2) \delta^{2}\right)$. By DMBI, such an end link adds the highest benefit if $k$ is as low as possible, i.e. if $k=1$. Note that any links received by $i_{c}$ and $i_{c}^{\prime}$ yield higher added benefits (they give strictly more added information, while its sponsor has no more ex-ante information). It follows the most stable diameter 3 network in which $i_{c}$ sponsors an end link, is where $k=1, i_{c}$ sponsors one end link and $i_{c}^{\prime}$ sponsors no end links (by the same arguments as above). Denote this most stable network of diameter 3 by $g^{3}$.

We now compare the stability of $g^{3}$ to that of $g^{4}$. The minimal added benefit of a link in $g^{3}$ is $\left(1+2 \delta+(n-3) \delta^{2}\right)-f\left(1+\delta+(n-3) \delta^{2}\right)$ which is strictly 
lower, by DMBI, than $f\left(1+2 \delta+(n-4) \delta^{2}+\delta^{3}\right)-f\left(1+\delta+(n-4) \delta^{2}+\delta^{3}\right)$. The reason is that $i$ in $g^{4}$ and $i_{c}$ in $g^{3}$ get the same increase in information through their end link $(\delta)$, but $i_{c}$ is without his end link better informed in $g^{3}$ than $i$ is without his end link in $g^{4}$.

Without further proof, Lemma 8 gives us the following result:

Proposition 4 Under DMBI, a gap in the diameters of the networks in $G^{S N N}$ can appear. In this case all SNN are either PSS (which have diameter 2), or they have a network diameter of at least $D^{*}$, with $D^{*} \geq 4$.

Propositions 3 and 4 suggest the following intuition. There are two forces that can drive a network to be stable, in giving the players incentives to sponsor costly links. First, players may sponsor links because ex ante they have few connections, but can access a lot of information by sponsoring a link to a wellinformed player. This effect is equally at work for CMBI, DMBI and IMBI, and is strongest in the PSS, explaining why overall this is the most stable network. Second, players may sponsor a link even though they already have many connections, if their marginal benefit of an additional connection is large. In the case of DMBI, the willingness of a player $i$ to sponsor a link $i j$ increases in the distance to the other players to which $i$ is connected ex ante. For this reason, large-diameter networks with outward pointing links may be stable on top of PSS networks. There is thus a gap in the diameters covered by the stable networks. In the case of IMBI, the willingness of a player $i$ to sponsor a link $i j$ decreases in the distance to the other players to which $i$ is connected ex ante. Thus, in the case of IMBI, the two different forces both lead to stable networks with a small diameter.

We derived the former results despite the fact that we did not identify the optimal recipient for each link. However, in Section 5 we derive a simple rule identifying the best-informed player in any component for a range of $\delta$ close to 1. We then show (Section 5.2) that all minimal connected networks satisfying both the balancing condition and the conditions from Proposition 1 are an SNN for some range of parameters. In this way, Proposition 1 and the balancing condition are sufficient conditions. Using the balancing condition, we establish our final two results in Section 5.3. First we derive the maximal diameter of candidate networks meeting the balancing condition. Second, we give a specific example of Proposition 4: in a network of 25 players (maximal diameter of balanced networks is 25) we obtain that the most stable network architecture (after the PSS) has a diameter of at least 7 , so $D^{*} \geq 7$.

In Section 5, the further assumptions made to make the characterization more detailed again impose weakly smaller decay. While this approach has its limits, it identifies the impact which decay has even when it would not lead to non-minimality of the network. Recall the well-known result that in the absence of decay only the CSS is a SNN. In this sense, our results, as well as Proposition 5.3 and 5.4 by Bala and Goyal (2000a), clearly show that the result under the absence of decay that only small-diameter networks are stable is not robust to 
the introduction of even a small level of decay. However from Bala and Goyal (2000a) and Hojman and Szeidl (2008) a reader can easily get the impression that with small decay the diameters of SNNs remain small. This impression would be false, as our example at the end of Section 5.3 shows.

\section{Small decay and no change in links: balancing condition}

As already pointed out, the necessary conditions of Section 3 for a given network to be a SNN include the condition that each link is received by the unique bestinformed player of the accessed set of players (Lemma 6). The problem is that the level of $\delta$ can affect which player is the best informed. Until now we did not present any results identifying the best-informed player in a set. In this section we do identify this player, but at the cost of a further restriction on $\delta$. In Section 5.1, we show that for high $\delta$, all networks which satisfy Lemma 6 satisfy a structural property which we call the balancing condition. We define the set of networks within $\mathcal{G}^{C A N}$ that meet this condition as $\mathcal{G}^{B A L}$, where $\mathcal{G}^{B A L}$ coincides with $\mathcal{G}^{C A N}(\delta)$ for all $\delta$ above a certain threshold $\delta_{B}(n)$. Intuitively, for small levels of decay, each link is sponsored "to the middle" of any accessed component, leaving no more players on one side than on the other sides combined (hence the label "balancing condition"). Thus, for $\delta$ large enough, all SNNs have to satisfy the properties of Proposition 1 and the balancing condition. So for small enough decay the balancing condition is a necessary condition for SNNs.

Section 5.2 is central to our paper, in showing the following sufficiency result: each minimal connected network which satisfies the four properties of Proposition 1 as well as the balancing condition is indeed a SNN for some positive range of parameters. In Section 5.3 we calculate the maximal diameter of networks in $\mathcal{G}^{B A L}$ as a function of $n$. An implication of the result is that there is no maximal diameter for SNNs provided that the population size can be chosen freely to accommodate that diameter. From the last two results it follows that that there are SNN with large diameters, although the diameter remains relatively small with respect to the population $\operatorname{size}^{21}$. Moreover, it allows us to provide an example of Proposition 4 with 25 players where the most-stable networks after the PSS have diameter at least 7 , i.e. $D^{*} \geq 7$.

\subsection{The balancing condition}

In this section we introduce the balancing condition. It roughly says that if $\delta$ is close enough to 1 and the network is a connected SNN, then the recipient of each link is "in the middle" of the group that the sponsor connects to through the link. More specifically, if he would sponsor the link to some neighbor of the recipient instead, there are less players that he would get closer to than players

\footnotetext{
${ }^{21}$ The minimum population size needed for a diameter $d$ candidate network which satisfies the balancing condition is exponentially increasing in the diameter.
} 
that he would get further away from. Put otherwise, the balancing condition says that in any accessed component, there should be no more players on one side of the recipient than on the other sides combined. To formalize this result, we will first define the balancing condition and then a new threshold decay level.

Definition 4 Network $g$ satisfies the balancing condition if for any $i j, \overline{j k} \in g$ we have that $\left|A_{i j}(g)\right|>2\left|A_{\overline{j k}}(g)\right|$ for all $\overline{j k} \in g$.

Definition 5 Let $\delta_{B}(n)$ be the lowest level of decay such that for all $\delta>\delta_{B}(n)$ all candidate networks which are SNN satisfy the balancing condition.

In Lemma 9 , we now show that $\delta_{B}(n)<1$. This will immediately give us the next Proposition which says that a given network satisfies Lemma 6 if and only if $\delta$ is large enough.

Lemma 9 For any $c>0, n \geq 3$, and $f(I)>0$ we have $\delta_{B}(n)<1$.

Proof. Denote by $i^{s}$ a player at distance $s$ from player $i$ in component $g_{A_{i i^{\prime}}(g)}$. Denote by $d_{\max }$ the maximal distance between $i$ and any other player in $g_{A_{i i^{\prime}}(g)}$. Finally, denote by $\mathcal{N}_{i}^{d}\left(g_{A_{i i^{\prime}}(g)}, i^{s}\right)$ the set of players at distance $d$ from player $i$ in network $g_{A_{i i^{\prime}}(g)}$ to which player $i^{s}$ gives access.

We now derive the condition under which a player $i$ sponsoring a link to $i^{\prime}$ in $g$ does not instead want to sponsor a link to any player $i^{2}$ at distance 2 from player $i$ in network $g_{A_{i i^{\prime}}(g)}$. The added information gain to player $i$ of sponsoring a link to player $i^{i^{\prime \prime}}=i^{1}$ in $g_{A_{i i^{\prime}}(g)}$ equals

$$
\sum_{d=1}^{d_{\max }} \delta^{d}\left|\mathcal{N}_{i}^{d}\left(g_{A_{i i^{\prime}}(g)}, i^{1}\right)\right| .
$$

Consider next the added information gain to player $i$ of sponsoring a link to a player $i^{2}$ at distance 2 from player $i$ in network $g_{A_{i i^{\prime}}(g)}$. The marginal information gain equals

$$
\sum_{d=1}^{d_{\max }}\left\{\delta^{d+1}\left[\left|\mathcal{N}_{i}^{d}\left(g_{A_{i i^{\prime}}(g)}, i^{1}\right)\right|-\left|\mathcal{N}_{i}^{d}\left(g_{A_{i i^{\prime}}(g)}, i^{2}\right)\right|\right]+\delta^{d-1}\left|\mathcal{N}_{i}^{d}\left(g_{A_{i i^{\prime}}(g)}, i^{2}\right)\right|\right\} .
$$

where $\left|\mathcal{N}_{i}^{r}\left(g_{A_{i i^{\prime}}(g)}, i^{r}\right)\right|=1$, and $\left|\mathcal{N}_{i}^{s}\left(g_{A_{i i^{\prime}}(g)}, i^{r}\right)\right|=0$ if $s<r$. This formula follows from observing that all players in set $\mathcal{N}_{i}^{d}\left(g_{A_{i i^{\prime}}(g)}, i^{2}\right)$ are one step closer than before by switching the link from $i i^{1}$ to $i i^{2}$, while the remainder of the players in $A_{i i^{1}}(g)$, namely $\left[\left|\mathcal{N}_{i}^{d}\left(g_{A_{i i^{\prime}}(g)}, i^{1}\right)\right|-\left|\mathcal{N}_{i}^{d}\left(g_{A_{i i^{\prime}}(g)}, i^{2}\right)\right|\right]$, are now one step further away. So $i$ strictly prefers to keep his link, rather than replacing it with a link to $i^{2}$ if the cost of getting one step further away from some agents $\left(\left[\left|\mathcal{N}_{i}^{d}\left(g_{A_{i i^{\prime}}(g)}, i^{1}\right)\right|-\left|\mathcal{N}_{i}^{d}\left(g_{A_{i i^{\prime}}(g)}, i^{2}\right)\right|\right]\right)$, outweighs the benefits of getting one 
step closer to some other agents $\left(\left|\mathcal{N}_{i}^{d}\left(g_{A_{i i^{\prime}}(g)}, i^{2}\right)\right|\right)$. This is the case if and only if

$$
\sum_{d=1}^{d_{\max }}\left\{\left[\delta^{d}-\delta^{d+1}\right]\left|\mathcal{N}_{i}^{d}\left(g_{A_{i i^{\prime}}(g)}, i^{1}\right)\right|+\left[\delta^{d+1}-\delta^{d-1}\right]\left|\mathcal{N}_{i}^{d}\left(g_{A_{i i^{\prime}}(g)}, i^{2}\right)\right|\right\}>0
$$

Define as $\delta_{B}<1$ the largest root of the polynomial on the left-hand side of (B) that is smaller than 1 . Another root of $(\mathrm{B})$ is $\delta=1$, reflecting the fact that in the absence of decay, it does not matter where the player connects. If the derivative with respect to $\delta$ of the left-hand side of (B) is negative at $\delta=1$, then a range of large $\delta$ s exists such that the player prefers to connect to $i^{1}$ rather than to any $i^{2}$. Taking the derivative of (B) with respect to $\delta$ and putting $\delta=1$, the condition becomes

$$
-\sum_{d=1}^{d_{\max }}\left|\mathcal{N}_{i}^{d}\left(g_{A_{i i^{\prime}}(g)}, i^{1}\right)\right|+2 \sum_{d=1}^{d_{\max }}\left|\mathcal{N}_{i}^{d}\left(g_{A_{i i^{\prime}}(g)}, i^{2}\right)\right|<0
$$

or

$$
\sum_{d=1}^{d_{\max }}\left|\mathcal{N}_{i}^{d}\left(g_{A_{i i^{\prime}}(g)}, i^{1}\right)\right|>2 \sum_{d=1}^{d_{\max }}\left|\mathcal{N}_{i}^{d}\left(g_{A_{i i^{\prime}}(g)}, i^{2}\right)\right|
$$

However, this only shows that the condition stated in the proposition is a necessary condition, as we have only derived a condition assuring that no player wants to reconnect to a player at distance 2 in the accessed component. To see that this condition is also a sufficient condition, consider a path $\left\{\overline{i^{1} i^{2}}, \overline{i^{2} i^{3}}, \ldots, \overline{i^{p} i^{p+1}}, \overline{i^{p+1} i^{p+2}}, \ldots, \overline{i^{z-1} i^{z}}\right\}$ in $g_{A_{i i^{\prime}}(g)}$, where player $i^{z}$ has no other links then $\overline{i^{z-1} i^{z}}$. Then by the given condition, for high $\delta$, player $i$ prefers connecting to $i^{p}$ rather than to $i^{p+1}$ if $\left|A_{i i^{p}}(g)\right|>2\left|A_{i^{p} i^{p+1}}(g)\right|$. Note now that $\left|A_{i i^{p}}(g)\right|=\left|A_{i i^{\prime}}(g)\right|$, whereas $\left|A_{\overline{i^{p} i^{p+1}}}(g)\right|<\left|A_{\overline{i^{\prime} j}}(g)\right|$. Thus, it follows from condition (B) that player $i$ prefers linking to any player $i^{p}$ rather than to player $i^{p+1}$ along the path. Condition (B) is thus sufficient.

The balancing condition translates Lemma 6 into a structural property of the network, provided that there is small enough decay. This is stated in the following Proposition.

Proposition 5 Let $\delta>\delta_{R}(c, n, f(I))$, and let $\delta>\delta_{B}(n)$. Then a given network satisfies Lemma 6 if and only if it meets the balancing condition.

Proof. This follows directly from the definition of the balancing condition, the definition of $\delta_{B}(n)$, and from Lemma 9. 
We call networks in $\mathrm{G}^{B A L}$ balanced networks, where

$$
\mathcal{G}^{B A L} \equiv\left\{g \in \mathcal{G}^{C A N} \mid g \text { satisfies the balancing condition }\right\} .
$$

As the necessary conditions characterizing $\mathrm{G}^{C A N}$ (Section 3) do not specify the best-informed player, it follows that $\mathrm{G}^{B A L} \subset \mathrm{G}^{C A N}$. It can be checked that all the examples in Figure 2 satisfy the balancing condition. The best-informed player in any accessed component can be considered as what sociologists call the player with the highest centrality in the component. Several concepts of centrality coexist in social network analysis (Freeman, 1978/1979). As can be seen in networks $(\mathrm{G})$ and (I) in Figure 2, the central player in our balanced networks need not be the player with the highest degree, so that our concept of centrality does not fit the network-analysis concept of degree centrality. Instead, the concept of centrality implied by the balancing condition bears resemblance to the network-analysis concept of betweenness centrality, which argues that the player with the most power in a component is the player that to the largest extent forms a bridge between the different players.

\subsection{Are balanced networks indeed SNNs?}

The main problem with having only necessary conditions for SNNs in a characterization is that the characterization may include networks which are not SNN. In this subsection we show that this problem does not apply to balanced networks. In other words: for each balanced network there exists some feasible positive range of parameters and some class of benefit functions such that this balanced network is in fact a SNN.

Proposition 6 Consider any network $g$ that satisfies the properties of Proposition 1 and the balancing condition. Then, for any CMBI and IMBI benefit function, levels of $\delta$ and $c$ exist such that $g$ is a SNN. For DMBI, a DMBI benefit function and levels of $\delta$ and $c$ exist such that $g$ is a $S N N$.

Proof. The proof consists of three steps. Steps (i) and (ii) together prove the results for CMBI and IMBI. Step (iii) proves the result for DMBI.

(i) For $g$ to be a SNN, it must be that every sponsor prefers to sponsor any subset of his sponsored links rather than not sponsoring them. Put otherwise, no sponsor should prefer to delete any of his sponsored links. As $\delta>0$, the average added benefit of sponsoring any subset of links is positive. It follows that we can find a small $c$ such that no sponsor wants to delete any links.

(ii) The added information gain of sponsoring any $k$ links is increasing in $\delta$, as more added information is obtained from any sponsored links the less decay there is. Also, ex ante information (before the $k$ links are sponsored) is larger the larger $\delta$. It follows that for CMBI and IMBI, the average added benefit of sponsoring any $k$ links is increasing in $\delta$. Given this fact, for any sufficiently low $c$ obtained from (i), we can increase $\delta$ until both $\delta>\delta_{M}(c, n, f(I))$ and $\delta>\delta_{B}(n)$, meaning that no player in $g$ wants to add links, nor replace links, 
while it continues to be the case that no sponsor wants to delete links in $g$. Hence $g$ is a SNN.

(iii) The minimal added benefit which any link can give in $g$ under DMBI is $f(1+(n-1) \delta)-f(1+(n-2) \delta)$. So for $c<f(1+(n-1) \delta)-f(1+(n-2) \delta)$ no player wants to delete links. However, for DMBI, while it continues to be true that the added information gain of sponsoring any $k$ links is increasing in $\delta$, any sponsor also has more ex ante information before sponsoring the links, so that it is possible that $f(1+(n-1) \delta)-f(1+(n-2) \delta)$ is decreasing in $\delta$. We provide a DMBI benefit function for which this is not the case. Let $f(I)=I^{y}$ with $0<y<1$. Then it can be checked that $f(1+(n-1) \delta)-f(1+(n-2) \delta)$ is increasing in $\delta .{ }^{22}$ It follows that if for $f(I)=I^{y}$ we take $c$ such that $c<$ $f(1+(n-1) \delta)-f(1+(n-2) \delta)$, no player in $g$ wants to delete links. If we further increase $\delta$ until both $\delta>\delta_{M}(c, n, f(I))$ and $\delta>\delta_{B}(n)$, by the above, for $f(I)=I^{y}$ it continues to be the case that no player wants to delete links.

Having shown that each balanced network is indeed a SNN for the right parameters, we are interested in the relationship between the maximal diameter of balanced networks and the population size. We investigate this in the following section.

\subsection{Maximal diameter of balanced networks}

In this section we derive the maximal diameter which a minimal connected SNN can have as a function of $n$, if decay is small enough. Before doing this, we present a lemma which specifies the minimum number of players that can be at a given distance from a sponsor $i$. We end the section by providing an example where the most stable networks are the PSS, and networks with either the maximal diameter minus one or the maximal diameter.

The balancing condition allows us to characterize the maximal-diameter SNN for all $n \geq 5$ (for smaller $n$, only stars are $\mathrm{SNN}$ ). We start by deriving a Lemma that we need to find the maximal-diameter SNN.

Lemma 10 Consider a balanced network $g$. Let link $i j \in g$ be such that it gives $i$ access to an end recipient at distance $d$ from player $i$. There are two cases. In Case 1, $i$ gets access to the end recipient through a link sponsored by $j$. In Case $2, i$ gets access to the end recipient through a link received by $j$.

1. If $i$ gets access to the end recipient at distance $d$ through a link $j k$, then in the component $g_{A_{i j}(g)}$ player $i$ has at least $\sum_{l=1}^{x+1} 2^{l}$ players at distance $(d-x)$ or larger from him, where $0 \leq x \leq(d-2)$.

${ }^{22} \frac{\partial}{\partial \delta}\left[(1+(n-1) \delta)^{y}-(1+(n-2) \delta)^{y}\right]>0 \Longleftrightarrow y(n-1)(1+(n-1) \delta)^{y-1}>y(n-2)(1+$ $(n-2) \delta)^{y-1}$. Canceling out $y$, and multiplying both sides by $(1+(n-1) \delta)(1+(n-2) \delta)$, the latter condition can be rewritten as $(n-1)(1+(n-2) \delta)(1+(n-1) \delta)^{y}>(n-2)(1+(n-$ $1) \delta)(1+(n-2) \delta)^{y}$. It is easy to see now that $(n-1)(1+(n-2) \delta)>(n-2)(1+(n-1) \delta)$. Further, given that $y>0,(1+(n-1) \delta)^{y}>(1+(n-2) \delta)^{y}$. It follows that the condition is valid. 
2. If $i$ gets access to the end recipient at distance $d$ through a link $k j$ (where $k j \neq i j)$, then in the component $g_{A_{i j}(g)}$, for $d \geq 3$, (a) player $i$ has at least $\sum_{l=1}^{x+1} 2^{l}$ players at distance $(d-x)$ or larger from him, where $0 \leq x \leq(d-4)$ (with $d \geq 4$ ); (b) player $i$ has at least $1+\sum_{l=1}^{d-3} 2^{l}$ players at distance 3 or larger in component $g_{A_{i j}(g)}$; and (c) player $i$ has at least $4+2 \sum_{l=1}^{d-3} 2^{l}$ players at distance 2 or larger in component $g_{A_{i j}(g)}$.

Proof. We first prove 1 . and 2(a), by induction. Note that by Lemma 7 , in $g_{A_{\overline{j k}}(g)}$, all links point away from $k$. If an end sponsor $m$ receives a link from a player $l$, then by Property 3 of Proposition $1, m$ should sponsor at least two links.

Let an end sponsor $m$ receiving a link from a player $l$ sponsor exactly two end links. Then, if player $l$ is himself a recipient, by the balancing condition (see Proposition 5), player $l$ 's links should point to at least 6 players at distance 1 or 2 from him, and at least 2 players at distance 2 from him.

Let player $l$ 's links point to exactly 6 players, at distance 1 or 2 from him, and let player $l$ himself receive a link from player $h$. Then, if player $h$ is himself a recipient, his links should point to at least 14 players at distance 1,2 or 3 from him, at least 6 players at distance 2 or 3 from him, and at least 2 players at distance 3 from him. And so forth.

We next prove $2(\mathrm{~b})$. This follows simply from the fact that there must be at least one player at distance 3 , and as already shown, at least $\sum_{l=1}^{d-3} 2^{l}$ players at distance 4 or larger.

In order to prove $2(\mathrm{c})$, note first that given the above, there is at least one player $k$ connected to player $j$ who gives access to at least $1+\sum_{l=1}^{d-3} 2^{l}$ players. This means that $\left|A_{\overline{j k}}(g)\right| \geq 2+\sum_{l=1}^{d-3} 2^{l}$, meaning that by the balancing condition, $\left|A_{i j}(g)\right|>4+2 \sum_{l=1}^{d-3} 2^{l}$

We now derive the maximal diameter networks that can be achieved for a particular range of $n$. In order to do this, we derive the minimal number of players $n$ that are needed to achieve a network of given diameter $D$. For this $n$, and for a range of populations just above it, this is then also the maximal diameter network that can be achieved. 
Proposition 7 A balanced network with even diameter $D, D \geq 4$, has at least $n=4+3 \sum_{l=1}^{D / 2-1} 2^{l-1}$ players. For $n=4+3 \sum_{l=1}^{D / 2-1} 2^{l-1}$, a diameter- $D$ network exists. ${ }^{23}$

Proof.

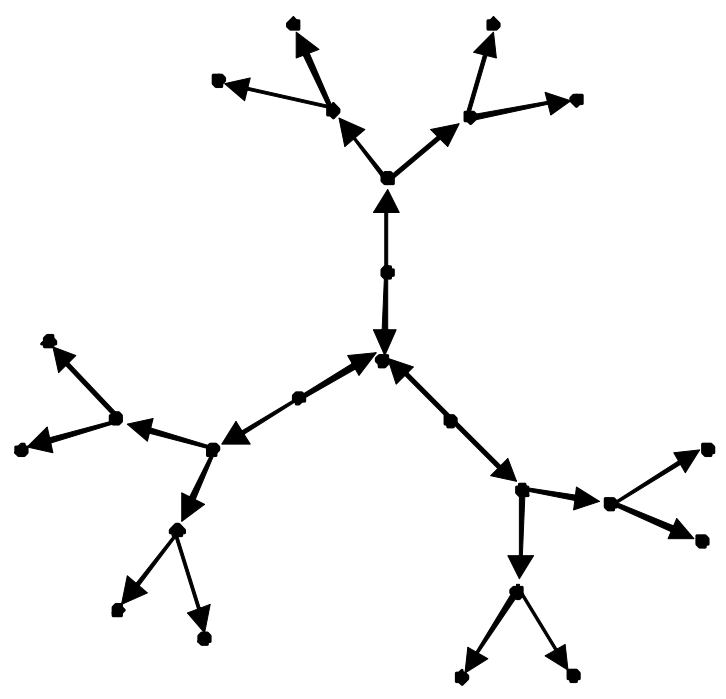

Figure 3: Maximal-diameter network for $\mathrm{n}=25$.

We first show that the minimal number of players needed to achieve a network with a multi-recipient characterizing player of even diameter $D \geq 4$ is the given $n$. By the definition of a multi-recipient player, at least two of the players at distance 1 from such a characterizing player must sponsor links towards him (though the characterizing player may sponsor links himself as well). Consider then one such sponsor of the multi-recipient characterizing player. In order to put an end recipient at distance $d$ from this sponsor using a minimal number of players, it should be that he accesses this end recipient through a link received by the multi-recipient characterizing player, and not through a link sponsored

\footnotetext{
${ }^{23}$ In the working paper version of this paper, we also derive the minimal number of players needed to achieve a diameter- $d$ network with odd diameter. This yields a set of levels of $n$ minimally needed to achieve any diameter- $d$ network. This then fully characterizes the maximal diameter that can be achieved for any given $n$, as for an $n_{x}$ not included in the set of levels of $n$, the maximal achievable diameter is then the diameter corresponding to the largest $n$ in the set of levels that is smaller than $n_{x}$.
} 
by him. This follows from Lemma 10 and from the fact that $4+2 \sum_{l=1}^{d-3} 2^{l}<\sum_{l=1}^{d-1} 2^{l}$. Thus, in order to construct, using a minimal number of players, a network with a multi-recipient characterizing player where two end recipients are at distance $d$ from each other, we can limit ourselves to networks where for each of these two end recipients, there is sponsor of the characterizing player accessing the end recipient through a link received by the characterizing player.

Consider in particular such a diameter- $D$ with two end recipients each at distance $D / 2$ of the multi-recipient characterizing player, and let us derive the minimal number of players necessary to construct such a network (we will show below that more players are needed to put the end recipients at different distances from the characterizing player and maintain diameter $D$ ). By Lemma 10 , sponsor $i$ (respectively $k$ ) of the characterizing player $j$ must give sponsor $k(i)$ access to at least $1+\sum_{l=1}^{D / 2-1} 2^{l-1}$ players (where $i(k)$ is included himself). This means that sponsor $k$ (respectively $i$ ) has this same number of players at distance 2 or more in the component that includes $i(k)$ and the links sponsored by $i(k)$. Applying the balancing condition now, it follows that $k(i)$ should have at least this same number of links at distance 2 or more in components to which characterizing player $j$ gives access, but to which $i(k)$ does not give access. It follows that $n=4+3 \sum_{l=1}^{D / 2-1} 2^{l-1}$ is the minimal number of players necessary to construct a diameter- $D$ with two end recipients at opposite sides each at distance $D / 2$ from the characterizing player.

To show that a balanced network with such a number of players indeed exists, consider a network where exactly three players sponsor a link to the multi-recipient characterizing player, and where each sponsor sponsors exactly two links. We call this the two-sponsor network. There are exactly $3 * 2^{l-2}$ players at distance $l$ from the characterizing player, with $2 \leq l \leq D / 2$, whose links point away from the characterizing player. Such a network indeed meets Property 3 of Proposition 1, and meets the balancing condition. An example is the network in Figure 3 for $n=25$ and $D=8$.

Next, we show that alternative diameter- $D$ networks with a multi-recipient characterizing player, where at one end of the network an end recipient is at distance smaller than $D / 2$ of the characterizing player, and at the other end of the network an end recipient is at distance larger than $D / 2$, use a larger number of players. By Lemma 10, an exponentially increasing number of players is needed to increase the maximal distance between the characterizing player and an end recipient. Thus decreasing the distance between the characterizing player and an end recipient on one side of the network and increasing this distance at the other side of the network while maintaining diameter $D$ is only possible when more players are used. 
Second, we show that with the given $n=4+3 \sum_{l=1}^{D / 2-1} 2^{l-1}$, one cannot construct a network with a non-recipient characterizing player that achieves a higher diameter than $D$. In order to show this, we show that, for even $D$, the minimal number of players needed to achieve a diameter- $D$ network with a non-recipient characterizing player is always at least as high as the minimal number of players needed to achieve a diameter- $d$ network with a multi-recipient characterizing player. Consider a diameter- $D$ network with a non-recipient characterizing player with two end recipients at distance $D$ from one another, and each at distance $D / 2$ from the characterizing player. In such a network, the characterizing player sponsors at least two links. By Lemma 10, the characterizing player has at least $2 \sum_{l=1}^{D / 2-1} 2^{l}$ players at distance 2 or more from him. Together with the minimum of two players at distance 1 , this means a minimum of $2+2 \sum_{l=1}^{D / 2-1} 2^{l}=2 \sum_{l=1}^{D / 2} 2^{l-1}$ players at distance 1 or more. Thus, to construct such a network, we need a minimum of $n=1+2 \sum_{l=1}^{D / 2} 2^{l-1}$ players. To show that a network using this number of players indeed exists, consider a symmetric network where there are exactly $2^{l}$ players at distance $l$ from the characterizing player, with $1 \leq l \leq D / 2$, and note that this network meets Property 3 of Proposition 1, and meets the balancing condition. By Lemma 10, decreasing the distance between the characterizing player and an end recipient at one side of characterizing player in order to increase the distance between the characterizing player and an end recipient on the other side of the characterizing player is only possible with the use of more players. It follows that $n=1+2 \sum_{l=1}^{D / 2} 2^{l-1}$ is the minimal number of players with which we can construct a diameter- $D$ network with a non-recipient characterizing player.

Finally, note that for $D \geq 4,1+2 \sum_{l=1}^{D / 2} 2^{l-1} \geq 4+3 \sum_{l=1}^{D / 2-1} 2^{l-1}$ (where equality is obtained only for $D=4$ ). It follows that to construct a network with even diameter $D \geq 4$, one needs at least $4+3 \sum_{l=1}^{D / 2-1} 2^{l-1}$ players.

We conclude that networks of any diameter can be balanced networks, provided that $n$ is large enough. Proposition 6 ensures that any such network can indeed be a SNN. Therefore SNNs may have any finitely large diameter, if the population size can be freely chosen. This result differs significantly from the small-diameter networks which were reported by earlier studies on this subject, namely the stars and interlinked stars (diameter 3) in Bala and Goyal (2000), and the PSS in Hojman and Szeidl (2008). 
We now combine the result that large-diameter networks can be SNN with the result from Proposition 4 that under DMBI, a gap in the diameters of the networks in $\mathcal{G}^{S N N}$ can appear. In Proposition 4, it is shown that under DMBI this gap may take such a form that all SNN either have diameter 2, or have diameter at least 4 . Knowing now that networks with a large diameter exist, it follows that this diameter gap may be even larger. Intuitively, sponsors have less ex ante information the larger the diameter of the network, suggesting that under DMBI the strictly most stable non-PSS balanced networks are the networks with the largest diameters.

We show that this intuition is valid for the network game with $n=25$ and DMBI, where for large $\delta$ the maximal-diameter architecture in Figure 3 $(D=8)$ is strictly more stable than any balanced network with diameter 6 or lower. As established before for, $c$ low enough and $\delta$ large enough any instability of a balanced network comes from the desire to delete a link. As a first step, we prove that the sponsor which is least willing to keep this link is the end sponsor with the least information. In a second step, we construct a diameter 6 network which has an end sponsor $i$ such that $i$ has the least amount of ex ante information over all $n$-player connected networks of diameter 6 or lower. Finally, in a third step, we show that player $i$ in this diameter- 6 network, in spite of his ex ante information being as small as it could be, still has more ex ante information than any end sponsor in the network of Figure 3.

Step 1. By Corollary 2, each non-PSS network contains at least one end sponsor. For $\delta=1$, in any non-PSS network, the crucial sponsor is clearly an end sponsor. It follows that this continues to be true for sufficiently large $\delta$. In the maximal-diameter network of Figure 3, for large $\delta$, given the symmetry of the network, each end sponsor is a crucial sponsor. For any end sponsor in this network, the ex ante information before sponsoring a single end link equals $\left(1+2 \delta+2 \delta^{2}+3 \delta^{3}+2 \delta^{4}+2 \delta^{5}+4 \delta^{6}+8 \delta^{7}\right)$. Step 2. The least possible ex ante information that an end sponsor can have in a network with diameter 6 or lower is when all players are at a distance as large as possible from the end sponsor. This is the case in a diameter-6 network where an end sponsor has ex ante information $\left(1+\delta+\delta^{2}+\delta^{3}+\delta^{4}+19 \delta^{5}\right)$, i.e. in a network that takes the form of a line connected to a star. Step 3. Finally, we show that for large $\delta$, $\left(1+\delta+\delta^{2}+\delta^{3}+\delta^{4}+19 \delta^{5}\right)>\left(1+2 \delta+2 \delta^{2}+3 \delta^{3}+2 \delta^{4}+2 \delta^{5}+4 \delta^{6}+8 \delta^{7}\right)$. The latter condition can be restated as the inequality $\left(1+\delta+2 \delta^{2}+\delta^{3}+4 \delta^{5}+8 \delta^{6}-17 \delta^{4}\right)<0$. As the left-hand side of this inequality is 0 for $\delta=1$, its first derivative with respect to $\delta$ valued at $\delta=1$ should be positive. The first-derivative of the lefthand side is $\left(1+4 \delta+3 \delta^{2}+20 \delta^{4}+48 \delta^{5}-68 \delta^{3}\right)$, which is indeed positive for $\delta=1$.

It follows that for $n=25$, under DMBI and with large enough $c$, the diameter gap can take such a form that all SNNs either have diameter 2 (the PSS), or have the maximal diameter (diameter 8, Figure 3) or the maximal diameter minus one (diameter 7 ). 


\section{Conclusion}

The purpose of this paper has been to investigate what happens when deviating from a standard network formation model without decay by introducing small levels decay. We wish to highlight three of the contributions of this paper. A first contribution has been to derive necessary conditions that minimal connected networks must have under decay in order to be strict Nash networks (SNN). We showed that only two types of minimal connected networks can be SNNs, namely networks with a unique characterizing player receiving multiple links, and networks with a unique characterizing player not receiving any links. Further, we showed that, with the exception of any links directly received by the characterizing player, the direction in which links are sponsored points away from the characterizing player. Moreover, each recipient of a link must either not sponsor any links, or must sponsor at least two links. It follows that, as long as the population is large enough, our necessary conditions do not impose any limit on the diameter of candidate equilibrium networks. Finally, for small enough decay any player sponsoring a link to a given component must sponsor a link "to the middle" of the accessed components, since for small decay, the node in the middle will also be the best-informed node. We called candidate equilibrium networks with this property balanced networks.

A second contribution of this paper was a sufficiency result, namely that any balanced network is indeed an SNN for some range of parameters.

A third contribution of this paper was to analyze the relative stability of different network architectures. A general result is that if any non-empty network is stable, then all periphery-sponsored stars are stable. This is because the characterizing player in this case has the highest possible quantity of information, and connecting to him is an all-or-nothing decision. Under constant marginal benefits of information, if any other non-empty networks are stable, then all networks meeting our necessary conditions are stable. Under increasing marginal benefits of information, the second-most stable networks are other star networks. Intuitively, it is the fact that the characterizing player already has a lot of information that under increasing marginal benefits of information still gives him an incentive to sponsor another link. Under decreasing marginal benefits of information, we showed that the second most stable network architecture (after the PSS) can have a diameter of at least $D^{*} \geq 4$, leading to a gap in the diameters covered by the SNN. For a particular example, we showed that this gap may be large, in that only the PSS and the networks with maximal diameter, or with diameter just below that, are stable. Intuitively, it is the fact that players have relatively little information in a large-diameter network that gives them strong incentives to sponsor their links.

Lacking from our analysis is the case of larger decay, where non-minimal networks may be SNN. Yet, it should be stressed that even for this case, our analysis characterizes those minimal networks that continue to be SNN under larger decay. The characterization of non-minimal networks is a different problem, and requires full attention in separate work. This is the subject of future research. 


\section{References}

Bala, V. and S. Goyal (2000a), "A noncooperative model of network formation", Econometrica 68(5), pp. 1181-1229.

Bala, V. and S. Goyal (2000b), "A strategic analysis of network reliability", Review of Economic Design 5(3), pp. 205-228.

Billand, P., C. Bravard and S. Sarangi (2006), "Heterogeneity in Nash Networks", Department of Economics Working Paper 2006-18, Louisiana State University.

Bloch, F. and B. Dutta (2009), "Communication networks with endogeneous link strength", Games and Economic Behavior 66(1), pp. 39-56.

Buechel, B. (2007), "Network formation with closeness incentives", Working Papers 398, Institute of Mathematical Economics, Bielefeld University.

Cohen, W.M. and D.A. Levinthal (1989), "Innovation and learning: the two faces of R\&D", The Economic Journal 99, pp 569-596.

Freeman, L.C. (1978/1979), "Centrality in social networks - conceptual clarification", Social Networks 1, 215-239.

Galeotti, A., S. Goyal and J. Kamphorst (2006), "Network formation with heterogeneous players", Games and Economic Behavior 54(2), pp. 353-372.

Goyal, S. and S. Joshi (2006), "Unequal connections", International Journal of Game Theory 34(3), pp 319-349.

Haller, H. and S. Sarangi (2005) "Nash networks with heterogeneous links", Mathematical Social Sciences 50(2), pp. 181-201.

Hojman, D. A. and A. Szeidl (2008), "Core and periphery in networks", Journal of Economic Theory 139(1), pp 295-309.

Kamphorst, J. and G. van der Laan (2007), "Network formation under heterogeneous costs: the multiple group model", International Game Theory Review 9(4), pp. 599-635.

Vergara-Caffarelli, F. (2004), "Non-cooperative network formation with network maintenance costs," Economics Working Papers ECO2004/18, European University Institute. 